APPLICATIONES MATHEMATICAE

25,2 (1998), pp. 221-252

J. RENCEA W OWICZ and W. M. Z A J A̧CZKOWSKI (Warszawa)

\title{
LOCAL EXISTENCE OF SOLUTIONS OF \\ THE MIXED PROBLEM FOR THE SYSTEM OF EQUATIONS OF IDEAL RELATIVISTIC HYDRODYNAMICS
}

Abstract. Existence and uniqueness of local solutions for the initialboundary value problem for the equations of an ideal relativistic fluid are proved. Both barotropic and nonbarotropic motions are considered. Existence for the linearized problem is shown by transforming the equations to a symmetric system and showing the existence of weak solutions; next, the appropriate regularity is obtained by applying Friedrich's mollifiers technique. Finally, existence for the nonlinear problem is proved by the method of successive approximations.

1. Introduction. In this paper we prove the local existence of solutions to the equations of ideal relativistic hydrodynamics which are the following system of conservation laws:

$$
T_{, x^{j}}^{i j}=0, \quad i, j=0,1,2,3,
$$

and

$$
\left(\delta u^{i}\right)_{x^{i}}=0,
$$

where the summation convention over repeated indices is assumed and

$$
T^{i j}=w u^{i} u^{j}+p g^{i j}
$$

is the energy-momentum tensor, and $g^{i j}$ is the space-time metric tensor of

1991 Mathematics Subject Classification: 35L60, 35Q75, 35A07, 35L65.

Key words and phrases: relativistic hydrodynamics, existence, initial-boundary value problem, system of hyperbolic equations of the first order, symmetrization. 
the form

$$
\left\{g^{i j}\right\}=\left[\begin{array}{cccc}
-1 & 0 & 0 & 0 \\
0 & 1 & 0 & 0 \\
0 & 0 & 1 & 0 \\
0 & 0 & 0 & 1
\end{array}\right]
$$

Moreover, $w=e+p$, where $w$ is the density of enthalpy, $e$ the density of the internal energy and $\delta$ the density of the fluid particles in a suitable system of coordinates in which the volume element does not move. We denote by $p$ the pressure and by $u=\left\{u^{i}\right\}_{i=0,1,2,3}$ the four-velocity: $u_{\alpha}=v_{\alpha} /(c \beta)$, $\alpha=1,2,3, u_{0}=-1 / \beta$, where $\beta=\sqrt{1-v^{2} / c^{2}}, c$ is the speed of light, $v^{2}=v_{1}^{2}+v_{2}^{2}+v_{3}^{2}$, where $v=\left(v_{1}, v_{2}, v_{3}\right)$ is the velocity vector, and $u^{i}=g^{i j} u_{j}$, $g^{i j}=g_{i j}, g^{i j} g_{j k}=\delta_{k}^{i}$.

In the above notation the energy-momentum tensor takes the form

$$
\begin{aligned}
& T_{\alpha \gamma}=w \frac{v_{\alpha} v_{\gamma}}{c^{2} \beta^{2}}+p \delta_{\alpha \gamma}, \quad \alpha, \gamma=1,2,3, \\
& T_{\alpha 0}=-w \frac{v_{\alpha}}{c \beta^{2}}, \quad T_{00}=\frac{w}{\beta^{2}}-p .
\end{aligned}
$$

We consider problem (1.1)-(1.2) for $t \in[0, T]$ and $x=\left(x_{1}, x_{2}, x_{3}\right) \in \Omega \subset$ $\mathbb{R}^{3}$, with initial and boundary conditions

$$
\begin{aligned}
& \left.(p, u, \delta)\right|_{t=0}=\left(p_{0}, u_{0}, \delta_{0}\right), \\
& \left.M z\right|_{\partial \Omega}=g\left(x^{\prime}, t\right),
\end{aligned}
$$

where $z=(p, u, \delta)$, and the matrix $M$ is defined in Section 4 .

To prove the existence of solutions to (1.1)-(1.2), we have to transform our problem to a symmetric hyperbolic system (2.2). We present this symmetrization in Section 2. In Section 3 we introduce the necessary spaces and norms; moreover, we rewrite the symmetric system (2.2) in the form (3.1) (with the initial-boundary conditions (1.6)-(1.7) suitably transformed).

In Section 4 we consider the linearized problem (3.1); first in 4(a) we prove the existence of solutions in a half-space, in 4(b) we obtain the regularity of solutions and in the last part of the section, using a partition of unity and a localized problem, we transform the results of 4(a) and 4(b) to the case of a bounded domain. Using the properties of the solutions obtained, we prove the existence and uniqueness of local solutions to the nonlinear problem (3.1) by the method of successive approximations in Section 5.

Finally, in Section 6 we specify the results of Sections 4 and 5 for problem (2.2). In Section 7 the barotropic case is considered.

To prove existence of solutions to problem (1.1), (1.2), (1.6), (1.7) we need to know that the form (6.1) is uniformly positive definite. To show it we choose a state equation (here $p=R \delta T$ ). This implies strong restrictions 
on the initial velocity (see Remark 6.1). In the barotropic case we do not have such restrictions so we can also consider near light motions.

2. Symmetrization. To symmetrize equations (1.1)-(1.2) we use considerations from [1], [2]. We have a system of conservation laws; now we write a new conservation law, which is a consequence of the old ones. (1.1) implies

$$
u_{i} \frac{\partial\left(w u^{k}\right)}{\partial x^{k}}+w u^{k} \frac{\partial u_{i}}{\partial x^{k}}+\frac{\partial p}{\partial x_{i}}=0 .
$$

Multiplying by $u^{i}$, summing over $i$ and using

$$
u^{i} u_{i}=-1, \quad u^{i} \frac{\partial u_{i}}{\partial x^{k}}=0
$$

we get

$$
-\frac{\partial\left(w u^{k}\right)}{\partial x^{k}}+u^{i} \frac{\partial p}{\partial x_{i}}=0
$$

which is equivalent to

$$
\frac{\partial}{\partial x^{k}}\left(\frac{w}{\delta} \delta u^{k}\right)-\frac{1}{\delta} \frac{\partial p}{\partial x^{k}} \delta u^{k}=0 .
$$

From this and (1.2) we obtain

$$
\delta u^{k}\left[\frac{\partial}{\partial x^{k}}\left(\frac{w}{\delta}\right)-\frac{1}{\delta} \frac{\partial p}{\partial x^{k}}\right]=0
$$

so using the thermodynamical identity, we can write

$$
T \delta u^{k} \frac{\partial}{\partial x^{k}}\left(\frac{s}{\delta}\right)=0
$$

where $s$ is the entropy. so

Finally, because $u^{k} s\left(-\frac{1}{\delta}\right) \frac{\partial \delta}{\partial x^{k}}=s \frac{\partial x^{k}}{\partial x^{k}}$ from (1.2), we get $T \frac{\partial}{\partial x^{k}}\left(s u^{k}\right)=0$,

$$
\frac{\partial}{\partial x^{k}}\left(s u^{k}\right)=0
$$

is a new conservation law.

We have shown that equations (1.1)-(1.3) are linearly dependent, that is, there exist functions $\lambda^{m}$ such that

$$
\lambda^{i} \frac{\partial T_{i}^{k}}{\partial x^{k}}+\lambda^{4} \frac{\partial\left(\delta u^{k}\right)}{\partial x^{k}}+\lambda^{5} \frac{\partial\left(s u^{k}\right)}{\partial x^{k}}=0
$$

for arbitrary functions $z^{j}\left(p, u^{1}, u^{2}, u^{3}, \delta\right)$, where $\lambda^{i}=u^{i}, i=0,1,2,3 ; \lambda^{4}=$ $(w-s T) / \delta, \lambda^{5}=T$ and $T=T(\delta, p), s=s(\delta, p)$. 
Equations (1.1)-(1.3) can be written in the form

$$
\partial_{z^{j}} q_{m}^{k}(z) \frac{\partial z^{j}}{\partial x^{k}}=0, \quad m=0,1, \ldots, 5,
$$

and multiplying by $\partial_{z^{\tau}} \lambda^{m}$ we obtain

$$
\partial_{z^{\tau}} \lambda^{m} \partial_{z^{j}} q_{m}^{k}(z) \frac{\partial z^{j}}{\partial x^{k}}=0 \Leftrightarrow A_{\tau j}^{k} \frac{\partial z^{j}}{\partial x^{k}}=0
$$

where the matrices $A^{k}(z)$ are symmetric (see [1], [2]).

The matrices $\partial_{z} q^{k}(z)$ take the form

$$
\begin{aligned}
& \partial_{z} q^{0}=\left(\begin{array}{ccccc}
1-\frac{1}{\beta^{2}}-\frac{1}{\beta^{2}} \frac{\partial e}{\partial p} & -2 u^{1} w & -2 u^{2} w & -2 u^{3} w & -\frac{1}{\beta^{2}} \frac{\partial e}{\partial \delta} \\
\frac{1}{\beta} u_{1}+\frac{1}{\beta} u_{1} \frac{\partial e}{\partial p} & \beta u_{1}^{2} w+\frac{w}{\beta} & \beta u_{1} u^{2} w & \beta u_{1} u^{3} w & \frac{u_{1}}{\beta} \frac{\partial e}{\partial \delta} \\
\frac{1}{\beta} u_{2}+\frac{1}{\beta} u_{2} \frac{\partial e}{\partial p} & \beta u_{2} u^{1} w & \beta u_{2}^{2} w+\frac{w}{\beta} & \beta u_{2} u^{3} w & \frac{u_{2}}{\beta} \frac{\partial e}{\partial \delta} \\
\frac{1}{\beta} u_{3}+\frac{1}{\beta} u_{3} \frac{\partial e}{\partial p} & \beta u_{3} u^{1} w & \beta u_{3} u^{2} w & \beta u_{3}^{2} w+\frac{w}{\beta} & \frac{u_{3}}{\beta} \frac{\partial e}{\partial \delta} \\
0 & \beta u^{1} \delta & \beta u^{2} \delta & \beta u^{3} \delta & \frac{1}{\beta} \\
-\frac{1}{\beta} \frac{\partial s}{\partial p} & \beta u^{1} s & \beta u^{2} s & \beta u^{3} s & \frac{1}{\beta} \frac{\partial s}{\partial \delta}
\end{array}\right), \\
& \partial_{z} q^{1}=\left(\begin{array}{ccccc}
-\frac{u^{1}}{\beta}-\frac{u^{1}}{\beta} \frac{\partial e}{\partial p} & -\beta u_{1}^{2} w-\frac{w}{\beta} & -\beta u^{1} u^{3} w & -\beta u^{1} u^{2} w & -\frac{u^{1}}{\beta} \frac{\partial e}{\partial \delta} \\
1+u_{1}^{2}+u_{1}^{2} \frac{\partial e}{\partial p} & 2 u_{1} w & 0 & 0 & u_{1}^{2} \frac{\partial e}{\partial \delta} \\
u^{1} u_{2}+u^{1} u_{2} \frac{\partial e}{\partial p} & u_{2} w & u^{1} w & 0 & u^{1} u_{2} \frac{\partial e}{\partial \delta} \\
u^{1} u_{3}+u^{1} u_{3} \frac{\partial e}{\partial p} & u_{3} w & 0 & u^{1} w & u^{1} u_{3} \frac{\partial e}{\partial \delta} \\
0 & \delta & 0 & 0 & u^{1} \\
u^{1} \frac{\partial s}{\partial p} & s & 0 & 0 & u^{1} \frac{\partial s}{\partial \delta}
\end{array}\right) \text {, } \\
& \partial_{z} q^{2}=\left(\begin{array}{ccccc}
-\frac{u^{2}}{\beta}-\frac{u^{2}}{\beta} \frac{\partial e}{\partial p} & -\beta u_{2} u_{1} w & -\beta u_{2}^{2} w-\frac{w}{\beta} & -\beta u_{3} u_{2} w & -\frac{u^{2}}{\beta} \frac{\partial e}{\partial \delta} \\
u^{2} u_{1}+u^{2} u_{1} \frac{\partial e}{\partial p} & u^{2} w & u_{1} w & 0 & u^{2} u_{1} \frac{\partial e}{\partial \delta} \\
1+u_{2}^{2}+u_{2}^{2} \frac{\partial e}{\partial p} & 0 & 2 u_{2} w & 0 & u_{2}^{2} \frac{\partial e}{\partial \delta} \\
u^{2} u_{3}+u^{2} u_{3} \frac{\partial e}{\partial p} & 0 & u_{3} w & u^{2} w & u^{2} u_{3} \frac{\partial e}{\partial \delta} \\
0 & 0 & \delta & 0 & u^{2} \\
u^{2} \frac{\partial s}{\partial p} & 0 & s & 0 & u^{2} \frac{\partial s}{\partial \delta}
\end{array}\right), \\
& \partial_{z} q^{3}=\left(\begin{array}{ccccc}
-\frac{u^{3}}{\beta}-\frac{u^{3}}{\beta} \frac{\partial e}{\partial p} & -\beta u_{3} u_{1} w & -\beta u^{3} u_{2} w & -\beta u_{3}^{2} w-\frac{w}{\beta} & -\frac{u^{3}}{\beta} \frac{\partial e}{\partial \delta} \\
u^{3} u_{1}+u^{3} u_{1} \frac{\partial e}{\partial p} & u^{3} w & 0 & u_{1} w & u^{3} u_{1} \frac{\partial e}{\partial \delta} \\
u^{3} u_{2}+u^{3} u_{2} \frac{\partial e}{\partial p} & 0 & u^{3} w & u_{2} w & u^{3} u_{2} \frac{\partial e}{\partial \delta} \\
1+u_{3}^{2}+u_{3}^{2} \frac{\partial e}{\partial p} & 0 & 0 & 2 u_{3} w & u_{3}^{2} \frac{\partial e}{\partial \delta} \\
0 & 0 & 0 & \delta & u^{3} \\
u^{3} \frac{\partial s}{\partial p} & 0 & 0 & s & u^{3} \frac{\partial s}{\partial \delta}
\end{array}\right) .
\end{aligned}
$$


The matrix $\partial_{z^{\tau}} \lambda^{m}$ has the form

$$
\left(\begin{array}{cccccc}
0 & 0 & 0 & 0 & \frac{\partial}{\partial p}\left(\frac{w-s T}{\delta}\right) & \frac{\partial}{\partial p} T \\
\beta u_{1} & 1 & 0 & 0 & 0 & 0 \\
\beta u_{2} & 0 & 1 & 0 & 0 & 0 \\
\beta u_{3} & 0 & 0 & 1 & 0 & 0 \\
0 & 0 & 0 & 0 & \frac{\partial}{\partial \delta}\left(\frac{w-s T}{\delta}\right) & \frac{\partial}{\partial \delta} T
\end{array}\right)
$$

so we get

$$
\begin{aligned}
& A^{0}=\left(\begin{array}{ccccc}
\frac{1}{\beta} \frac{\partial s}{\partial p} \frac{\partial T}{\partial p} & \beta u_{1} & \beta u_{2} & \beta u_{3} & \frac{1}{\beta} \frac{\partial s}{\partial p} \frac{\partial T}{\partial \delta} \\
\beta u_{1} & -\beta u_{1}^{2} w+\frac{w}{\beta} & -\beta u_{1} u^{2} w & -\beta u_{1} u^{3} w & 0 \\
\beta u_{2} & -\beta u_{2} u^{1} w & -\beta u_{2}^{2} w+\frac{w}{\beta} & -\beta u_{2} u^{3} w & 0 \\
\beta u_{3} & -\beta u_{3} u^{1} w & -\beta u_{3} u^{2} w & -\beta u_{3}^{2} w+\frac{w}{\beta} & 0 \\
\frac{1}{\beta} \frac{\partial s}{\partial p} \frac{\partial T}{\partial \delta} & 0 & 0 & 0 & \frac{1}{\beta} \frac{\partial T}{\partial \delta}\left(\frac{\partial s}{\partial \delta}-\frac{s}{\delta}\right)
\end{array}\right), \\
& A^{1}=\left(\begin{array}{ccc}
u^{1} \frac{\partial s}{\partial p} \frac{\partial T}{\partial p} & 1 & 0 \\
1 & -\beta^{2} u_{1}^{3} w+u^{1} w & -\beta^{2} u_{1}^{2} u^{2} w \\
0 & -\beta^{2} u_{1}^{2} u_{2} w & -\beta^{2} u^{1} u_{2}^{2} w+u^{1} w \\
0 & -\beta^{2} u_{1}^{2} u_{3} w & -\beta^{2} u^{1} u^{2} u_{3} w \\
u^{1} \frac{\partial s}{\partial p} \frac{\partial T}{\partial \delta} & 0 & 0
\end{array}\right. \\
& 0 \\
& -\beta^{2} u_{1}^{2} u^{3} w \\
& -\beta^{2} u^{1} u_{2} u^{3} w \\
& -\beta^{2} u^{1} u_{3}^{2} w+u^{1} w \\
& 0 \quad u^{1} \frac{\partial T}{\partial \delta}\left(\frac{\partial s}{\partial \delta}-\frac{s}{\delta}\right) \\
& A_{00}^{k}=u^{k} \frac{\partial s}{\partial p} \frac{\partial T}{\partial p}, \quad A_{\alpha 0}^{0}=\beta u_{\alpha}, \quad A_{\alpha 0}^{k^{\prime}}=\delta_{\alpha}^{k^{\prime}}, \quad \alpha, k^{\prime}, \gamma=1,2,3, \\
& A_{40}^{k}=u^{k} \frac{\partial s}{\partial p} \frac{\partial T}{\partial \delta}, \quad A_{\alpha \gamma}^{k}=-\beta^{2} u^{k} u_{\alpha} u^{\gamma} w+u^{k} w \delta_{\alpha}^{\gamma}, \\
& A_{4 \alpha}^{k}=0, \quad A_{44}^{k}=u^{k} \frac{\partial T}{\partial \delta}\left(\frac{\partial s}{\partial \delta}-\frac{s}{\delta}\right) .
\end{aligned}
$$

Now we consider the following symmetric system:

$$
A^{k}(z)\left(\begin{array}{l}
p, x^{k} \\
u_{, x^{k}}^{1} \\
u_{, x^{k}}^{2} \\
u_{, x^{k}}^{3} \\
\delta_{, x^{k}}
\end{array}\right)=0 ; \quad k=0,1,2,3,
$$


or, because $x_{0}=c t$,

$$
A^{0}(z) z_{t}+c \sum_{i=1}^{3} A^{i}(z) z_{x^{i}}=0
$$

3. Notations. In the next sections we will use the following norms, spaces and notations.

We will consider initial-boundary value problems in $\Omega^{T}=\Omega \times[0, T]$ where $\Omega \subset \mathbb{R}^{3}, x \in \Omega, t \in[0, T]$. We write

$$
D_{t, x}^{\gamma}=\frac{\partial^{\gamma^{0}}}{\partial t^{\gamma_{0}}} \frac{\partial^{\gamma_{1}}}{\partial x_{1}^{\gamma_{1}}} \frac{\partial^{\gamma_{2}}}{\partial x_{2}^{\gamma_{2}}} \frac{\partial^{\gamma_{3}}}{\partial x_{3}^{\gamma_{3}}}, \quad|\gamma|=\gamma_{0}+\gamma_{1}+\gamma_{2}+\gamma_{3},
$$

and we denote by $H^{s}\left(\Omega^{T}\right)$ the Sobolev space with the norm

$$
\|u\|_{H^{s}\left(\Omega^{T}\right)}=\left(\sum_{|\gamma| \leq s} \int_{0}^{T} \int_{\Omega}\left|D_{t, x}^{\gamma} u\right|^{2} d x d t\right)^{1 / 2}=\|u\|_{s, 2, \Omega^{T}} .
$$

Similarly, we introduce $H^{s}(\Omega)$ and $H^{s}\left(\partial \Omega^{T}\right)$ with norms \|\|$_{s, 2, \Omega}$ and \|\|$_{s, 2, \partial \Omega^{T}}$. We will use $L_{p}\left(\Omega^{T}\right)$ and $L_{p}(\Omega)$ with norms \|\|$_{p, \Omega^{T}}$ and \|\|$_{p, \Omega}$, respectively.

For $\alpha \in \mathbb{R}$ we denote by $H_{\alpha}^{s}\left(\Omega^{T}\right)$ the weighted Sobolev space, the closure of $C^{s}\left(\Omega^{T}\right)$ in the norm

$$
\|u\|_{H_{\alpha}^{s}\left(\Omega^{T}\right)}=\|u\|_{s, \Omega^{T}, \alpha}=\left(\sum_{|\gamma| \leq s} \int_{0}^{T} \int_{\Omega}\left|D_{t, x}^{\gamma} u\right|^{2} e^{-2 \alpha t} d x d t\right)^{1 / 2}
$$

so we obtain $L_{2, \alpha}\left(\Omega^{T}\right)=H_{\alpha}^{0}\left(\Omega^{T}\right)$ with \|\|$_{L_{2, \alpha}\left(\Omega^{T}\right)}=\|\|_{\Omega^{T}, \alpha}$.

Let

$$
u \in L_{\infty}^{s}\left(0, T ; H^{i}(\Omega)\right) \Leftrightarrow \underset{t \in[0, T]}{\operatorname{ess} \sup }\left\|\frac{\partial^{s}}{\partial t^{s}} u(t)\right\|_{i, 2, \Omega}<\infty .
$$

Then we define

$$
\Pi_{k}^{l}\left(\Omega^{T}\right)=\bigcap_{i=k}^{l} L_{\infty}^{l-i}\left(0, T ; H^{i}(\Omega)\right)
$$

with $\|u\|_{\Pi_{k}^{l}\left(\Omega^{T}\right)}=\|u\|_{l, k, \infty, \Omega^{T}}$. Finally, we introduce $\Gamma_{0}^{l}(\Omega)$ by

$$
\|u\|_{\Gamma_{0}^{l}(\Omega)}=|u|_{l, 0, \Omega}=\left(\sum_{|\gamma| \leq l} \int_{\Omega}\left|D_{t, x}^{\gamma} u\right|^{2} d x\right)^{1 / 2} .
$$

Furthermore, $\stackrel{\circ}{\Gamma}_{0}^{l}, \stackrel{\circ}{H}_{\alpha}^{s}$ denote the sets of functions in the respective spaces vanishing on the boundary $\partial \Omega ;|u|$ is the Euclidean norm. 
To simplify the following considerations, in Sections 4 and 5 we will consider the mixed problem

$$
\begin{aligned}
& L u \equiv E(t, x, u) u_{t}+\sum_{i=1}^{3} A_{i}(t, x, u) u_{x_{i}}=F(t, x), \\
& \left.M\left(t, x^{\prime}, u\right) u\right|_{\partial \Omega}=g\left(t, x^{\prime}\right), \quad x^{\prime} \in \partial \Omega, \\
& \left.u\right|_{t=0}=u_{0}(x),
\end{aligned}
$$

where $u$ takes values in $\mathbb{R}^{m}, x \in \Omega \subset \mathbb{R}^{3}, t \in[0, T], E, A_{i}$ are real $m \times m$ matrices, the values of $u$ lie in an open domain $G$ and the values of the initial data $u_{0}$ belong to an open subset $G_{0}$ such that $\bar{G}_{0} \subset G$. Next, assuming $u:=z \in \mathbb{R}^{5}, z=\left(p, u^{1}, u^{2}, u^{3}, \delta\right)$ we will formulate results for problem (2.2) with initial and boundary conditions (1.6), (1.7).

4(a) The existence of solutions for the linearized equations in a halfspace. In this part we shall consider the linearized problem (3.1) in the half-space $x_{1}>0$ :

$$
\begin{aligned}
& L u=E(t, x) u_{t}+\sum_{i=1}^{3} A_{i}(t, x) u_{x_{i}}=F(t, x), \\
& \left.M\left(t, x^{\prime}\right) u\right|_{x_{1}=0}=g\left(t, x^{\prime}\right), \\
& \left.u\right|_{t=0}=u_{0},
\end{aligned}
$$

where $x=\left(x_{1}, x^{\prime}\right)$ and we assume that $\Omega=\left\{x \in \mathbb{R}^{3}: x_{1}>0\right\}, \partial \Omega=\{x \in$ $\left.\mathbb{R}^{3}: x_{1}=0\right\}$. In part (c) we shall obtain results for a bounded domain $\Omega$, using a partition of unity.

Lemma 4.1. (1) Let $E, A_{i}, i=1, \ldots, 3$, be symmetric matrices and

$$
E u \cdot u \geq \alpha_{0} u^{2}, \quad \alpha_{0}>0 .
$$

Let $\bar{n}$ be the unit outward vector normal to $\partial \Omega$ and assume $-A_{\bar{n}}=A_{1}$ has eigenvalues $\lambda_{\mu}$, where $\lambda_{\mu}^{+}, \mu=1, \ldots, k$, and $\lambda_{\mu}^{-}, \mu=k+1, \ldots, m$, are respectively the positive and negative ones. Suppose that

$$
\min _{\mu} \min _{\Omega^{T}}\left|\lambda_{\mu}\right| \geq c_{0}>0
$$

and

$$
\max _{\nu \in\{1, \ldots, k\}} \max _{\partial \Omega^{T}} \lambda_{\nu}^{+}\left(x^{\prime}, t\right) \leq c_{1},
$$

where $c_{0}, c_{1}$ are constants.

(2) Let $\gamma_{\mu}^{+}, \gamma_{\mu}^{-}$be orthonormal eigenvectors of the matrix $-A_{\bar{n}}$, corresponding to the eigenvalues $\lambda_{\mu}^{+}, \lambda_{\mu}^{-}$. Assume that the matrix $M\left(t, x^{\prime}\right)$ has 
the form

$$
\begin{aligned}
M= & \sum_{\mu, \nu=1}^{k} \alpha_{\mu \nu}\left(t, x^{\prime}\right) \gamma_{\mu}^{+}\left(t, x^{\prime}\right) \gamma_{\nu}^{+}\left(t, x^{\prime}\right) \\
& +\sum_{\mu=1}^{k} \sum_{\nu=k+1}^{m} \beta_{\mu \nu}\left(t, x^{\prime}\right) \gamma_{\mu}^{+}\left(t, x^{\prime}\right) \gamma_{\nu}^{-}\left(t, x^{\prime}\right)
\end{aligned}
$$

where
(a) $\max _{\partial \Omega^{T}}\left|\alpha_{\mu \nu}^{-1}\left(t, x^{\prime}\right)\right| \leq \delta_{0}^{-1}$,
(b) $\max _{\partial \Omega^{T}}\left|\beta_{\mu \nu}\left(t, x^{\prime}\right)\right| \leq \beta_{0}$,
(c) $\left(c_{0}+c_{1}\right) \delta_{0}^{-2} \beta_{0}^{2} \leq \frac{1}{2} c_{0}$,

and $\delta_{0}, \beta_{0}$ are constants.

(3) Let $\widetilde{L}=\left(E, A_{1}, A_{2}, A_{3}\right), \widetilde{L}, M \in \Pi_{0}^{3}\left(\Omega^{T}\right)$ and suppose that $\alpha$ satisfies

$$
|\widetilde{L}|_{3,0, \infty, \Omega^{T}}<\alpha \alpha_{0} / 2
$$

and

$$
\sup _{\Omega^{T}}|E| \leq c_{2}, \quad \text { where } c_{2} \text { is a constant. }
$$

Then, for every $u \in C^{\infty}\left(\Omega^{T}\right)$ and $t \leq T$ we have the estimate

$$
\begin{gathered}
\alpha_{0} e^{-2 \alpha t} \int_{\Omega} u^{2} d x+\frac{\alpha \alpha_{0}}{2} \int_{\Omega^{t}} u^{2} e^{-2 \alpha s} d x d s+\frac{\alpha_{0}}{2} \int_{\partial \Omega^{t}} u^{2} e^{-2 \alpha s} d x^{\prime} d s \\
\leq\left(c_{0}+c_{1}\right) \delta_{0}^{-2} \int_{\partial \Omega^{t}}|M u|^{2} e^{-2 \alpha s} d x^{\prime} d s \\
\quad+\frac{2}{\alpha \alpha_{0}} \int_{\Omega^{t}}|L u|^{2} e^{-2 \alpha s} d x d s+\left.c_{2} \int_{\Omega} u^{2} d x\right|_{t=0} .
\end{gathered}
$$

Proof. Multiplying (4.1) 1 by $u e^{-2 \alpha t}$ and integrating by parts in $\Omega$, we obtain

$$
\begin{aligned}
& \frac{d}{d t} e^{-2 \alpha t} \int_{\Omega} E u^{2} d x+2 \alpha e^{-2 \alpha t} \int_{\Omega} E u^{2} d x+e^{-2 \alpha t} \int_{\partial \Omega} A_{n} u^{2} d x^{\prime} \\
& -e^{-2 \alpha t} \int_{\Omega}\left(\sum_{i=1}^{3} A_{i, x_{i}}+E_{t}\right) u^{2} d x-2 e^{-2 \alpha t} \int_{\Omega} L u \cdot u d x=0 .
\end{aligned}
$$

Integrating (4.10) from 0 to $t$, using (4.2) and (4.8) we get

$$
\alpha_{0} e^{-2 \alpha t} \int_{\Omega} u^{2} d x+2 \alpha \alpha_{0} \int_{\Omega^{t}} u^{2} e^{-2 \alpha s} d x d s+\int_{\partial \Omega^{t}} A_{n} u^{2} e^{-2 \alpha s} d x^{\prime} d s
$$




$$
\begin{aligned}
\leq & \int_{\Omega^{t}}\left(\sum_{i=1}^{3} A_{i, x_{i}}+E_{s}\right) u^{2} e^{-2 \alpha s} d x d s \\
& +2 \int_{\Omega^{t}} L u \cdot u e^{-2 \alpha s} d x d s+\left.c_{2} \int_{\Omega} u^{2} d x\right|_{t=0} .
\end{aligned}
$$

From (4.7) we get

$$
\max _{\Omega^{t}}\left(\left|E_{t}\right|+\sum_{i=1}^{3}\left|A_{i, x_{i}}\right|\right) \leq 2 c|\widetilde{L}|_{3,0, \infty, \Omega^{T}} \leq \alpha \alpha_{0}
$$

so using the Young inequality (with $\varepsilon=\sqrt{2 /\left(\alpha \alpha_{0}\right)}$ ) in (4.11) we have

$$
\begin{array}{r}
\alpha_{0} e^{-2 \alpha t} \int_{\Omega} u^{2} d x+\frac{\alpha \alpha_{0}}{2} \int_{\Omega^{t}} u^{2} e^{-2 \alpha s} d x d s+\int_{\partial \Omega^{t}} A_{n} u^{2} e^{-2 \alpha s} d x^{\prime} d s \\
\leq \frac{2}{\alpha \alpha_{0}} \int_{\Omega^{t}}|L u|^{2} e^{-2 \alpha s} d x d s+\left.c_{2} \int_{\Omega} u^{2} d x\right|_{t=0} .
\end{array}
$$

We have to consider the boundary term. From (2),

$$
\begin{gathered}
u=\sum_{\mu=1}^{k} c_{\mu} \gamma_{\mu}^{+}+\sum_{\mu=k+1}^{m} c_{\mu} \gamma_{\mu}^{-}=u^{\prime}+u^{\prime \prime} \quad \text { where } c_{\mu}=u \gamma_{\mu}, \\
u^{\prime}=\sum_{\mu=1}^{k} c_{\mu} \gamma_{\mu}^{+}, \quad \text { so } \quad\left|u^{\prime}\right|^{2}=\sum_{\mu=1}^{k} c_{\mu}^{2}, \quad\left|u^{\prime \prime}\right|^{2}=\sum_{\mu=k+1}^{m} c_{\mu}^{2},
\end{gathered}
$$

and

$$
-A_{\bar{n}} u^{2}=\sum_{\mu=1}^{k} \lambda_{\mu}^{+} c_{\mu}^{2}+\sum_{\mu=k+1}^{n} \lambda_{\mu}^{-} c_{\mu}^{2} .
$$

Using this and (4.3), (4.4) we get, from (4.12),

$$
\begin{aligned}
\alpha_{0} e^{-2 \alpha t} \int_{\Omega} u^{2} d x+ & \frac{\alpha \alpha_{0}}{2} \int_{\Omega^{t}} u^{2} e^{-2 \alpha s} d x d s \\
& +c_{0} \int_{\partial \Omega^{t}}\left|u^{\prime \prime}\right|^{2} e^{-2 \alpha s} d x^{\prime} d s \\
\leq & c_{1} \int_{\partial \Omega^{t}}\left|u^{\prime}\right|^{2} e^{-2 \alpha s} d x^{\prime} d s \\
& +\frac{2}{\alpha \alpha_{0}} \int_{\Omega^{t}}|L u|^{2} e^{-2 \alpha s} d x d s+\left.c_{2} \int_{\Omega} u^{2} d x\right|_{t=0} .
\end{aligned}
$$


Now, to express $\left|u^{\prime}\right|^{2}=\sum_{\mu=1}^{k} c_{\mu}^{2}$ by $|M u|^{2}$, we consider

$$
M u=\sum_{\mu, \nu=1}^{k} \alpha_{\mu \nu} \gamma_{\mu}^{+} c_{\nu}^{+}+\sum_{\mu=1}^{k} \sum_{\nu^{\prime}=k+1}^{m} \beta_{\mu \nu^{\prime}} \gamma_{\mu}^{+} c_{\nu^{\prime}}^{-}=\sum_{\mu=1}^{k} g_{\mu} \gamma_{\mu}^{+}
$$

so

$$
g_{\mu}=\sum_{\nu=1}^{k} \alpha_{\mu \nu} c_{\nu}^{+}+\sum_{\nu^{\prime}=k+1}^{m} \beta_{\mu \nu^{\prime}} c_{\nu^{\prime}}^{-}
$$

and this implies

$$
c_{\nu}^{+}=\sum_{\mu=1}^{k} \alpha_{\mu \nu}^{-1} g_{\mu}-\sum_{\nu^{\prime}=k+1}^{m} \sum_{\mu=1}^{k} \alpha_{\mu \nu}^{-1} \beta_{\mu \nu^{\prime}} c_{\nu^{\prime}}^{-} .
$$

Adding $c_{0} \int_{\partial \Omega^{t}}\left|u^{\prime}\right|^{2} e^{-2 \alpha s} d x^{\prime} d s$, using (4.6) and the last expression, we obtain

$$
\begin{aligned}
\alpha_{0} e^{-2 \alpha t} \int_{\Omega} u^{2} d x+\frac{\alpha \alpha_{0}}{2} \int_{\Omega^{t}} u^{2} e^{-2 \alpha s} d x d s+c_{0} \int_{\partial \Omega^{t}} u^{2} e^{-2 \alpha s} d x^{\prime} d s \\
\leq\left(c_{0}+c_{1}\right) \delta_{0}^{-2} \int_{\partial \Omega^{t}}|M u|^{2} e^{-2 \alpha s} d x^{\prime} d s \\
\quad+\frac{2}{\alpha \alpha_{0}} \int_{\Omega^{t}}|L u|^{2} e^{-2 \alpha s} d x d s \\
\quad+\left(c_{0}+c_{1}\right) \delta_{0}^{-2} \beta_{0}^{2} \int_{\partial \Omega^{t}}\left|u^{\prime \prime}\right|^{2} e^{-2 \alpha s} d x^{\prime} d s+\left.c_{2} \int_{\Omega} u^{2} d x\right|_{t=0} .
\end{aligned}
$$

Finally, from (4.14) and (4.6)(c) we have (4.9).

To prove the existence of solutions to (4.1) we have to split it into a Cauchy problem and a boundary value problem. Let $\chi \in C_{0}^{\infty}(-\delta, \delta)$; we assume that a solution of (4.1) has the form $u=\chi u_{1}+u_{2}$, where

$$
L u_{1}=0,\left.\quad u_{1}\right|_{t=0}=u_{0},
$$

and

$$
L u_{2}=F-E u_{1} \frac{\partial}{\partial t} \chi,\left.\quad M u_{2}\right|_{\partial \Omega}=g,\left.\quad u_{2}\right|_{t=0}=0 .
$$

Further, introducing $w_{1}=u_{1}-\widetilde{u}_{0}$ (where $\widetilde{u}_{0}$ denotes an extension of $u_{0}$ to the half-space $t>0)$ we get, from (4.15),

$$
L w_{1}=-L \widetilde{u}_{0},\left.\quad w_{1}\right|_{t=0}=0 .
$$

We define the formally adjoint operator $L^{(*)}$ by

$$
L^{(*)}=-E \partial_{t}-\sum_{i=1}^{3} A_{i} \partial_{x_{i}}-E_{t}-\sum_{i=1}^{3} A_{i, x_{i}}
$$


so we have the identity

$$
\left(L w_{1}, v_{1}\right)_{\Omega^{T}}=\left(w_{1}, L^{(*)} v_{1}\right)_{\Omega^{T}}
$$

for all $w_{1}, v_{1} \in C_{0}^{\infty}\left(\Omega^{T}\right)$ with $\left.w_{1}\right|_{t=0}=0$ and $\left.v_{1}\right|_{t=T}=0$.

Next, for such $w_{1}, v_{1}$ we obtain by (4.9) the following estimates:

$$
\begin{array}{rl}
\alpha_{0} e^{-2 \alpha t} \int_{\Omega} w_{1}^{2} d x+\frac{\alpha \alpha_{0}}{2} \int_{\Omega^{t}} w_{1}^{2} e^{-2 \alpha s} & d x d s \\
\leq & \frac{2}{\alpha \alpha_{0}} \int_{\Omega^{t}}\left|L w_{1}\right|^{2} e^{-2 \alpha s} d x d s
\end{array}
$$

and (with time travelling backward)

$$
\alpha_{0} e^{2 \alpha t} \int_{\Omega} v_{1}^{2} d x+\frac{\alpha \alpha_{0}}{2} \int_{\Omega^{t}} v_{1}^{2} e^{2 \alpha s} d x d s \leq \frac{2}{\alpha \alpha_{0}} \int_{\Omega^{t}}\left|L^{(*)} v_{1}\right|^{2} e^{2 \alpha s} d x d s
$$

Now we use the following (see [3]).

THEOREM 4.1. Let $\mathcal{L}$ denote the space of square integrable functions on $\Omega^{T}, D_{L}$ the domain of $L$ consisting of $u \in C^{\infty}\left(\Omega^{T} \cup \partial \Omega^{T}\right)$ which satisfy the boundary (initial) condition, and $D_{L^{(*)}}$ the domain of $L^{(*)}$ of those $v \in C^{\infty}\left(\Omega^{T} \cup \partial \Omega^{T}\right)$ which satisfy the adjoint boundary (initial) condition. If there exists a constant $c$ such that

$$
c\|u\| \leq\|\bar{L} u\|, \quad c\|v\| \leq\left\|\bar{L}^{(*)} v\right\|,
$$

for $u \in D_{\bar{L}}$ and $v \in D_{\bar{L}^{(*)}}$, then $\bar{L}$ and $\bar{L}^{(*)}$ map their domains one-to-one onto $\mathcal{L}$.

From this theorem and inequalities (4.20), (4.21) we get:

LEMma 4.2. There exists a unique weak solution $u_{1}$ of (4.15) such that $w_{1} \in \stackrel{\circ}{L}_{2, \alpha}\left(\Omega^{T}\right)$.

Now we are looking for solutions of problem (4.16). For the adjoint $L^{(*)}$ we obtain the identity

$$
(L u, v)=\left(u, L^{*} v\right)+\left(A_{\bar{n}} u, v\right)=\left(u, L^{(*)} v\right)-\left(A_{1} u, v\right)
$$

where $u, v \in C_{0}^{1}(\Omega \times \mathbb{R})$.

We can find the boundary matrix $M^{*}$ for the adjoint problem from $\left(A_{1} u, v\right)=0$ for $u \in \operatorname{ker} M$ and $v \in \operatorname{ker} M^{*}$ (see [10], [11]). Let $F, g=0$ for $t<0$ and $t>T$. Then we consider (4.16) in $\Omega \times \mathbb{R}$ and we can prove, similarly to $(4.9)$,

$$
\begin{aligned}
& \frac{\alpha \alpha_{0}}{2} \int_{\Omega \times \mathbb{R}} u_{2}^{2} e^{-2 \alpha s} d x d s+\frac{c_{0}}{2} \int_{\partial \Omega \times \mathbb{R}} u_{2}^{2} e^{-2 \alpha s} d x^{\prime} d s \\
\leq & \left(c_{0}+c_{1}\right) \delta_{0}^{-2} \int_{\partial \Omega \times \mathbb{R}}\left|M u_{2}\right|^{2} e^{-2 \alpha s} d x^{\prime} d s+\frac{2}{\alpha \alpha_{0}} \int_{\Omega \times \mathbb{R}}\left|L u_{2}\right|^{2} e^{-2 \alpha t} d x d s .
\end{aligned}
$$


We have to obtain an estimate for the adjoint problem. If we take $L^{(*)}, M^{*}$ instead of $L, M$ and we assume that the time is travelling backward, then we can prove (in the same way as Lemma 4.1):

Lemma 4.3. Assume that (1) and (3) of Lemma 4.1 hold. Let

$$
\begin{aligned}
M^{*}= & \sum_{\mu, \nu=k+1}^{m} \alpha_{\mu \nu}^{*}\left(t, x^{\prime}\right) \gamma_{\mu}^{-}(t, x) \gamma_{\nu}^{-}\left(t, x^{\prime}\right) \\
& +\sum_{\mu=k+1}^{m} \sum_{\nu=1}^{k} \beta_{\mu \nu}^{*}\left(t, x^{\prime}\right) \gamma_{\mu}^{-}\left(t, x^{\prime}\right) \gamma_{\nu}^{+}\left(t, x^{\prime}\right),
\end{aligned}
$$

with

$$
\max _{\partial \Omega^{T}}\left|\alpha_{\mu \nu}^{*-1}\right| \leq \delta_{0}^{-1}, \quad \max _{\partial \Omega^{T}}\left|\beta_{\mu \nu}^{*}\right| \leq \gamma_{0}, \quad\left(c_{0}+c_{4}\right) \delta_{0}^{-2} \gamma_{0}^{2} \leq c_{0} / 2 .
$$

Moreover, let

$$
\max _{\nu \in\{1, \ldots, m\}} \max _{\partial \Omega \times \mathbb{R}}\left|\lambda_{\nu}^{-}\left(x^{\prime}, t\right)\right| \leq c_{4} .
$$

Then for $v_{2} \in C_{0}^{\infty}(\Omega \times \mathbb{R}) \cap L_{2,-\alpha}(\Omega \times \mathbb{R})$ we obtain

$$
\begin{aligned}
& \frac{\alpha \alpha_{0}}{2} \int_{\Omega \times \mathbb{R}} v_{2}^{2} e^{2 \alpha s} d x d s+\frac{c_{0}}{2} \int_{\partial \Omega \times \mathbb{R}} v_{2}^{2} e^{2 \alpha s} d x^{\prime} d s \\
\leq & \left(c_{0}+c_{4}\right) \delta_{0}^{-2} \int_{\partial \Omega \times \mathbb{R}}\left|M^{*} v_{2}\right|^{2} e^{2 \alpha s} d x^{\prime} d s+\frac{2}{\alpha \alpha_{0}} \int_{\Omega \times \mathbb{R}}\left|L^{*} v_{2}\right|^{2} e^{2 \alpha s} d x d s .
\end{aligned}
$$

Now, by (4.23), (4.26) and Theorem 4.1 we have

LEMMA 4.4. Let $g \in L_{2, \alpha}(\partial \Omega \times \mathbb{R})$ with $\left.g\right|_{t<0}=\left.g\right|_{t>T}=0$ and $F \in$ $L_{2, \alpha}(\Omega \times \mathbb{R})$ with $\left.F\right|_{t<0}=\left.F\right|_{t>T}=0$. Let the assumptions of Lemmas 4.1 and 4.3 be satisfied. Then there exists a unique solution $u_{2} \in L_{2, \alpha}(\Omega \times \mathbb{R})$ of (4.16) such that $\left.u_{2}\right|_{\partial \Omega} \in L_{2, \alpha}(\partial \Omega \times \mathbb{R})$.

In Lemmas 4.2 and 4.4 we can obtain strong solutions, using the technique of mollifiers (see [6], [12]) with respect to $\left(x^{\prime}, t\right)$ where $x=\left(x_{1}, x^{\prime}\right)$. Then we have the sequence $u_{\varepsilon}=J_{\varepsilon} u=j_{\varepsilon} * u$ (the operator $J_{\varepsilon}$ is the mollifier) and from the properties of $J_{\varepsilon}$ we have the convergences

$$
\begin{array}{ll}
u_{\varepsilon} \rightarrow u & \text { in } L_{2}\left(\Omega^{T}\right), \\
L u_{\varepsilon} \rightarrow L u=F & \text { in } L^{2}\left(\Omega^{T}\right), \\
M u_{\varepsilon} \rightarrow M u=g & \text { in } L^{2}\left(\partial \Omega^{T}\right),
\end{array}
$$

and $u_{\varepsilon}$ is continuous up to the boundary.

Now for $u=\chi u_{1}+u_{2}$ we formulate

Theorem 4.2. Let $u_{0} \in H^{1}(\Omega),\left.u_{0}\right|_{\partial \Omega}=0, \widetilde{L} \in H_{\alpha}^{1}\left(\Omega^{T}\right)$ and $F \in$ $L_{2, \alpha}\left(\Omega^{T}\right), g \in L_{2, \alpha}\left(\partial \Omega^{T}\right)$. Let the assumptions of Lemmas 4.1 and 4.3 be 
satisfied. Then there exists a unique strong solution $u$ of problem (4.1) in the half-space $\Omega$ such that $u \in L_{2, \alpha}\left(\Omega^{T}\right) \cap L_{2, \alpha}\left(\partial \Omega^{T}\right) \cap L_{\infty}\left(0, T ; \Gamma_{0}^{1}(\Omega)\right)$ and (4.9) holds.

4(b) Regularity of solutions. To prove the existence of solutions of (3.1) we have to use the method of successive approximations; so we need more regular solutions of (4.1) such that $u \in H^{3}\left(\Omega^{T}\right)$. Since $u \in L_{2, \alpha}\left(\Omega^{T}\right)$ we have to use mollifiers to derive the regularity of $u$. Let $u_{\delta}=j_{\delta} * u=J_{\delta} u$, where $j(t, x) \in C_{0}^{\infty}\left(\mathbb{R}^{1} \times \mathbb{R}^{n}\right), j \geq 0, \int j(t, x) d x d t=1$ and $j_{\delta}(t, x)=$ $\delta^{-n-1} j(t / \delta, x / \delta)$. We consider the problems

$$
\begin{aligned}
& L D_{t, x^{\prime}}^{s} u_{\delta}=D_{t, x^{\prime}}^{s} L u_{\delta}+\left(L D_{t, x^{\prime}}^{s} u_{\delta}-D_{t, x^{\prime}}^{s} L u_{\delta}\right), \\
& \left.M D_{t, x^{\prime}}^{s} u_{\delta}\right|_{x_{1}=0}=D_{t, x^{\prime}}^{s} M u_{\delta}+\left(M D_{t, x^{\prime}}^{s} u_{\delta}-D_{t, x^{\prime}}^{s} M u_{\delta}\right), \\
& \left.D_{t, x^{\prime}}^{s} u_{\delta}\right|_{t=0}=\left.D_{t, x^{\prime}}^{s} u_{\delta}\right|_{t=0},
\end{aligned}
$$

for $s=1,2,3$, where

$$
\begin{aligned}
D_{t, x^{\prime}}^{s} u & =\sum_{|\gamma|=s} \frac{\partial^{\gamma_{0}}}{\partial t^{\gamma_{0}}} \frac{\partial^{\gamma_{2}}}{\partial x_{2}^{\gamma_{2}}} \frac{\partial^{\gamma_{3}}}{\partial x_{3}^{\gamma_{3}}} u, \quad|\gamma|=\gamma_{0}+\gamma_{2}+\gamma_{3}, \\
L u_{\delta} & =(L u)_{\delta}-\left[(L u)_{\delta}-L u_{\delta}\right] \\
& =(L u)_{\delta}-C_{\delta} u \quad\left(C_{\delta} u \text { is called the commutator }\right) .
\end{aligned}
$$

Lemma 4.5. Assume that (1)-(3) of Lemma 4.1 hold, $M \in H_{\alpha}^{3}\left(\partial \Omega^{T}\right)$, $g \in H_{\alpha}^{3}\left(\partial \Omega^{T}\right), u_{0} \in H^{3}(\Omega)$ and $F \in H_{\alpha}^{3}\left(\Omega^{T}\right)$. Set

$$
a=|\widetilde{L}|_{3,0, \infty, \Omega^{t}}, \quad b=|M|_{3,0, \infty, \Omega^{T}}+\|M\|_{3, \partial \Omega^{t}, \alpha}
$$

for $t \leq T$. Then there exist polynomials $p_{0}(a, b), p_{s}(a, b), q_{s}(a, b), 1 \leq s \leq 3$, such that the solution of problem (4.1) satisfies the following estimate:

$$
\begin{aligned}
\alpha_{0}|u|_{s, 0, \Omega}^{2} e^{-2 \alpha t}+\frac{\alpha \alpha_{0}}{4} & \|u\|_{s, \Omega^{t}, \alpha}^{2}+\frac{c_{0}}{2}\|u\|_{s, \partial, \Omega^{t}, \alpha}^{2} \\
\leq & p_{s}(a, b)\left[|L u|_{s, \Omega^{t}, \alpha}^{2}+\left.|L u|_{s-1,0, \Omega}^{2}\right|_{t=0}\right] \\
& +q_{s}(a, b)\|M u\|_{s, \partial \Omega^{t}, \alpha}^{2}+\left.c_{2}|u|_{s, 0, \Omega}^{2}\right|_{t=0}
\end{aligned}
$$

where

$$
\alpha \text { satisfies } p_{0}(a, b) \leq \alpha \alpha_{0} \text {. }
$$

Moreover, there exist polynomials $r$ such that

$$
\left.|u|_{s, 0, \Omega}^{2}\right|_{t=0} \leq r\left(\left.|\widetilde{L}|_{s-1,0, \Omega}\right|_{t=0},\left.|L u|_{s-1,0, \Omega}\right|_{t=0},\left\|u_{0}\right\|_{s, 2, \Omega}\right) .
$$

Proof. For $|s|=1$ and problem (4.27) we have by (4.9), 


$$
\begin{aligned}
\alpha_{0} e^{-2 \alpha t} \int_{\Omega}\left|D_{t, x^{\prime}}^{1} u_{\delta}\right|^{2} d x & +\frac{\alpha \alpha_{0}}{2} \int_{\Omega^{t}}\left|D_{t, x^{\prime}}^{1} u_{\delta}\right|^{2} e^{-2 \alpha s} d x d s \\
& +\frac{c_{0}}{2} \int_{\partial \Omega^{t}}\left|D_{t, x^{\prime}}^{1} u_{\delta}\right| e^{-2 \alpha s} d x^{\prime} d s \\
\leq & \frac{2}{\alpha \alpha_{0}} \int_{\Omega^{t}}\left|D_{t, x^{\prime}}^{1} L u_{\delta}\right|^{2} e^{-2 \alpha s} d x d s \\
& +\frac{2}{\alpha \alpha_{0}} \int_{\Omega^{t}}\left|L D_{t, x^{\prime}}^{1} u_{\delta}-D_{t, x^{\prime}}^{1} L u_{\delta}\right|^{2} e^{-2 \alpha s} d x d s \\
& +c_{3} \int_{\partial \Omega^{t}}\left|D_{t, x^{\prime}}^{1} g_{\delta}^{\prime}\right|^{2} e^{-2 \alpha s} d x^{\prime} d s \\
& +c_{3} \int_{\partial \Omega^{t}}\left|M D_{t, x^{\prime}}^{1} u_{\delta}-D_{t, x^{\prime}}^{1} M u_{\delta}\right|^{2} e^{-2 \alpha s} d x^{\prime} d s \\
& +\left.c_{2} \int_{\Omega}\left|D_{t, x^{\prime}}^{1} u_{\delta}\right|^{2} d x\right|_{t=0}
\end{aligned}
$$

where

$$
c_{3}=\left(c_{0}+c_{1}\right) \delta_{0}^{-2}, \quad g_{\delta}^{\prime}=M u_{\delta} .
$$

We have to estimate the second and fourth terms on the right-hand side of (4.31). We can write

$$
\begin{aligned}
\int_{\Omega^{t}}\left(L D_{t, x^{\prime}}^{1} u_{\delta}-D_{t, x^{\prime}}^{1} L u_{\delta}\right)^{2} e^{-2 \alpha s} d x d s & \leq \int_{\Omega^{t}}\left|D_{t, x^{\prime}}^{1} \widetilde{L}\right|^{2}\left|D_{t, x}^{1} u_{\delta}\right|^{2} e^{-2 \alpha s} d x d s \\
& \leq c a^{2} \int_{\Omega^{t}}\left|D_{t, x}^{1} u_{\delta}\right|^{2} e^{-2 \alpha s} d x d s .
\end{aligned}
$$

Because

$$
D_{x_{1}}^{1} u_{\delta}=A_{1}^{-1}\left[L u_{\delta}-E u_{\delta t}-A^{\prime} u_{\delta x^{\prime}}\right], \quad A^{\prime} u_{\delta x^{\prime}}=\sum_{i=2}^{3} A_{i} u_{\delta x_{i}}
$$

and $\operatorname{det} A_{1} \geq c_{0}^{m},\left|A_{1}^{-1}\right| \leq c c_{0}^{-m} a^{m-1}$ we get

$$
\int_{\Omega^{t}}\left|D_{x_{1}}^{1} u_{\delta}\right|^{2} e^{-2 \alpha s} d x d s \leq c a^{2(m-1)}\left(\left\|L u_{\delta}\right\|_{0, \Omega^{t}, \alpha}^{2}+a^{2}\left\|u_{\delta}\right\|_{1, \Omega^{t}, \alpha}^{\prime 2}\right)
$$

(the prime denotes that the derivative $D_{x_{1}}$ does not appear), so finally

$$
\begin{aligned}
& \int_{\Omega^{t}}\left|L D_{t, x^{\prime}}^{1} u_{\delta}-D_{t, x^{\prime}}^{1} L u_{\delta}\right|^{2} e^{-2 \alpha s} d x d s \\
& \leq c a^{2 m}\left\|L u_{\delta}\right\|_{0, \Omega^{t}, \alpha}^{2}+c a^{2}\left(a^{2 m}+1\right)\left\|u_{\delta}\right\|_{1, \Omega^{t}, \alpha}^{2} .
\end{aligned}
$$

For the boundary term we have 


$$
\begin{aligned}
\int_{\partial \Omega^{t}}\left|M D_{t, x^{\prime}}^{1} u_{\delta}-D_{t, x^{\prime}}^{1} M u_{\delta}\right| e^{-2 \alpha s} d x^{\prime} d s \\
\quad \leq \int_{\partial \Omega^{t}}\left|D_{t, x^{\prime}}^{1} M\right|^{2} u_{\delta}^{2} e^{-2 \alpha s} d x^{\prime} d s \\
\quad \leq c b^{2} \int_{\partial \Omega^{t}}\left|u_{\delta}\right|^{2} e^{-2 \alpha s} d x d s \leq c b^{2} \int_{0}^{t} e^{-2 \alpha s} \int_{\Omega}\left|D_{x}^{1} u_{\delta}\right|^{2} d x d s \\
\quad \leq c b^{2} \int_{0}^{t} e^{-2 \alpha s} \int_{\Omega}\left(\left|D_{x_{1}}^{1} u_{\delta}\right|^{2}+\left|D_{x^{\prime}}^{1} u_{\delta}\right|^{2}+\left|D_{t}^{1} u_{\delta}\right|^{2}\right) d x d s \\
\quad \leq c b^{2}\left[\left\|u_{\delta}\right\|_{1, \Omega^{t}, \alpha}^{2}+\left\|D_{x_{1}}^{1} u_{\delta}\right\|_{0, \Omega^{t}, \alpha}^{2}\right] \\
\leq c b^{2}\left\|L u_{\delta}\right\|_{0, \Omega^{t}, \alpha}^{2}+c b^{2}\left(a^{2 m}+1\right)\left\|u_{\delta}\right\|_{1, \Omega^{t}, \alpha}^{2} .
\end{aligned}
$$

Assuming

$$
\frac{\alpha \alpha_{0}}{4} \geq c\left[\frac{2}{\alpha \alpha_{0}} a^{2}\left(a^{2 m}+1\right)+b^{2} c_{3}\left(a^{2 m}+1\right)\right]
$$

we obtain from (4.9), (4.31), (4.34) and (4.35),

$$
\begin{aligned}
& \alpha_{0}\left|u_{\delta}\right|_{1,0, \Omega}^{\prime 2} e^{-2 \alpha t}+\frac{\alpha \alpha_{0}}{4}\left\|u_{\delta}\right\|_{1, \Omega^{t}, \alpha}^{\prime 2}+\frac{c_{0}}{2}\left\|u_{\delta}\right\|_{1, \partial \Omega^{t}, \alpha}^{\prime 2} \\
& \quad \leq c \widetilde{p}_{1}(a, b)\left\|L u_{\delta}\right\|_{1, \Omega^{t}, \alpha}^{2}+c \widetilde{q}_{1}(a, b)\left\|M u_{\delta}\right\|_{1, \partial \Omega^{t}, \alpha}^{2}+\left.c_{2}\left|u_{\delta}\right|_{1,0, \Omega}^{\prime 2}\right|_{t=0}
\end{aligned}
$$

where $\widetilde{p}_{1}, \widetilde{q}_{1}$ are polynomials.

Using (4.32) we have

$$
\left\|u_{\delta x_{1}}\right\|_{0,2, \Omega}^{2} \leq c a^{2(m-1)}\left(\left\|L u_{\delta}\right\|_{0,2, \Omega}^{2}+a^{2}\left\|u_{\delta}\right\|_{1,0, \Omega}^{2}\right)
$$

so finally from (4.33), (4.37) and (4.36),

$$
\begin{aligned}
\alpha_{0}\left|u_{\delta}\right|_{1,0, \Omega}^{2} e^{-2 \alpha t}+\frac{\alpha \alpha_{0}}{4} & \left\|u_{\delta}\right\|_{1, \Omega^{t}, \alpha}^{2}+\frac{c_{0}}{2}\left\|u_{\delta}\right\|_{1, \partial \Omega^{t}, \alpha}^{2} \\
\leq & p_{1}(a, b)\left(\left\|L u_{\delta}\right\|_{1, \Omega^{t}, \alpha}^{2}+\left\|L u_{\delta}\right\|_{0,2, \Omega}^{2}\right) \\
& \quad+q_{1}(a, b)\left\|M u_{\delta}\right\|_{1, \partial \Omega^{t}, \alpha}^{2}+\left.c_{2}\left|u_{\delta}\right|_{1,0, \Omega}^{2}\right|_{t=0}
\end{aligned}
$$

where $p_{1}, q_{1}$ are polynomials.

Using the convergence $u_{\delta} \rightarrow u$ in $H^{1}$, and $L u_{\delta}=F_{\delta}-C_{\delta} u \rightarrow F=L u$ in $H^{1}$ (because $C_{\delta} u \rightarrow 0$ in $L^{2}$ and $H^{1}$ for sufficiently regular $\widetilde{L}$ ) we obtain

$$
\begin{aligned}
\alpha_{0}|u|_{1,0, \Omega}^{2} e^{-2 \alpha t}+\frac{\alpha \alpha_{0}}{4} & \|u\|_{1, \Omega^{t}, \alpha}^{2}+\frac{c_{0}}{2}\|u\|_{1, \partial \Omega^{t}, \alpha}^{2} \\
\leq & p_{1}(a, b)\left(\|L u\|_{1, \Omega^{t}, \alpha}^{2}+\|L u\|_{0,2, \Omega}^{2}\right) \\
& \quad+q_{1}(a, b)\|M u\|_{1, \partial \Omega^{t}, \alpha}^{2}+\left.c_{2}|u|_{1,0, \Omega}^{2}\right|_{t=0}
\end{aligned}
$$

so we have (4.28) for $s=1$. 
Let us consider the case $s=2$. We have, using (4.9) to (4.27),

$$
\begin{aligned}
\alpha_{0} e^{-2 \alpha t} \int_{\Omega}\left|D_{t, x^{\prime}}^{2} u_{\delta}\right|^{2} d x & +\frac{\alpha \alpha_{0}}{2} \int_{\Omega^{t}}\left|D_{t, x^{\prime}}^{2} u_{\delta}\right|^{2} e^{-2 \alpha s} d x d s \\
& +\frac{c_{0}}{2} \int_{\partial \Omega^{t}}\left|D_{t, x^{\prime}}^{2} u_{\delta}\right|^{2} e^{-2 \alpha s} d x^{\prime} d s \\
\leq & \frac{2}{\alpha \alpha_{0}} \int_{\Omega^{t}}\left|D_{t, x^{\prime}}^{2} L u_{\delta}\right|^{2} e^{-2 \alpha s} d x d s \\
& +\frac{2}{\alpha \alpha_{0}} \int_{\Omega^{t}}\left|L D_{t, x^{\prime}}^{2} u_{\delta}-D_{t, x^{\prime}}^{2} L u_{\delta}\right|^{2} e^{-2 \alpha s} d x d s \\
& +c_{3} \int_{\partial \Omega^{t}}\left|D_{t, x^{\prime}}^{2} g_{\delta}^{\prime}\right|^{2} e^{-2 \alpha s} d x^{\prime} d s \\
& +c_{3} \int_{\partial \Omega^{t}}\left|M D_{t, x^{\prime}}^{2} u_{\delta}-D_{t, x^{\prime}}^{2} M u_{\delta}\right|^{2} e^{-2 \alpha s} d x^{\prime} d s \\
& +\left.c_{2} \int_{\Omega}\left|D_{t, x^{\prime}}^{2} u_{\delta}\right|^{2} d x\right|_{t=0} .
\end{aligned}
$$

As before, we estimate

$$
\begin{aligned}
\int_{\Omega^{t}} \mid L D_{t, x^{\prime}}^{2} & u_{\delta}-\left.D_{t, x^{\prime}}^{2} L u_{\delta}\right|^{2} e^{-2 \alpha s} d x d s \\
& \leq \int_{\Omega^{t}}\left(\left|D_{t, x^{\prime}}^{2} \widetilde{L}\right|^{2}\left|D_{t, x^{\prime}}^{1} u_{\delta}\right|^{2}+\left|D_{t, x^{\prime}}^{1} \widetilde{L}\right|^{2}\left|D_{t, x^{\prime}}^{1} D_{t, x}^{1} u_{\delta}\right|^{2}\right) e^{-2 \alpha s} d x d s \\
& \leq c a^{2}\left(\left\|u_{\delta}\right\|_{1, \Omega^{t}, \alpha}^{2}+\int_{\Omega^{t}}\left|D_{t, x^{\prime}}^{1} D_{t, x^{\prime}}^{1} u_{\delta}\right|^{2} e^{-2 \alpha s} d x d s\right),
\end{aligned}
$$

$$
\begin{aligned}
& \text { (4.41) } \quad \int_{\Omega^{t}}\left|D_{x_{1}}^{1} D_{t, x^{\prime}}^{1} u_{\delta}\right|^{2} e^{-2 \alpha s} d x d s \\
& \leq c a^{2(m-1)}\left[\left\|L u_{\delta}\right\|_{1, \Omega^{t}, \alpha}^{2}+a^{2}\left(\left\|u_{\delta}\right\|_{1, \Omega^{t}, \alpha}^{\prime 2}+\left\|D_{t, x^{\prime}}^{2} u_{\delta}\right\|_{0, \Omega^{t}, \alpha}^{2}\right)\right], \\
& (4.42) \quad \int_{\Omega^{t}}\left|D_{x_{1}}^{1} D_{x_{1}}^{1} u_{\delta}\right|^{2} e^{-2 \alpha s} d x d s \\
& \leq c a^{2(m-1)}\left[\left\|L u_{\delta}\right\|_{1, \Omega^{t}, \alpha}^{2}+a^{2}\left(\left\|u_{\delta}\right\|_{1, \Omega^{t}, \alpha}^{2}+\left\|D_{x_{1}}^{1} D_{t, x^{\prime}}^{1} u_{\delta}\right\|_{0, \Omega^{t}, \alpha}^{2}\right)\right] \\
& \leq c\left[\left(a^{2(m-1)}+a^{4(m-1)}\right)\left\|L u_{\delta}\right\|_{1, \Omega^{t}, \alpha}^{2}\right. \\
& \left.\quad+\left(a^{2 m}+a^{4 m}\right)\left\|u_{\delta}\right\|_{1, \Omega^{t}, \alpha}^{2}+a^{4 m}\left\|D_{t, x^{\prime}}^{2} u_{\delta}\right\|_{0, \Omega^{t}, \alpha}^{2}\right]
\end{aligned}
$$

where (4.41), (4.42) are obtained by differentiating (4.32) with respect to 
$\left(t, x^{\prime}\right)$ and $x_{1}$, respectively. Hence

$$
\begin{aligned}
& \int_{\Omega^{t}}\left|L D_{t, x^{\prime}}^{2} u_{\delta}-D_{t, x^{\prime}}^{2} L u_{\delta}\right|^{2} e^{-2 \alpha s} d x d s \\
\leq & c a^{2}\left[\left(1+a^{2 m}\right)\left(\left\|u_{\delta}\right\|_{1, \Omega^{t}, \alpha}^{2}+\left\|D_{t, x^{\prime}}^{2} u_{\delta}\right\|_{0, \Omega^{t}, \alpha}^{2}\right)+a^{2(m-1)}\left\|L u_{\delta}\right\|_{1, \Omega^{t}, \alpha}^{2}\right] .
\end{aligned}
$$

For the boundary term we have

$$
\begin{aligned}
\int_{\partial \Omega^{t}} \mid M D_{t, x^{\prime}}^{2} u_{\delta} & -\left.D_{t, x^{\prime}}^{2} M u_{\delta}\right|^{2} e^{-2 \alpha s} d x^{\prime} d s \\
& \leq \int_{\partial \Omega^{t}}\left(\left|D_{t, x^{\prime}}^{2} M u_{\delta}\right|^{2}+\left|D_{t, x^{\prime}}^{1} M\right|^{2}\left|D_{t, x^{\prime}}^{1} u_{\delta}\right|^{2}\right) e^{-2 \alpha s} d x^{\prime} d s \\
& \leq c b^{2} \int_{\partial \Omega^{t}}\left(\left|u_{\delta}\right|^{2}+\left|D_{t, x^{\prime}}^{1} u_{\delta}\right|^{2}\right) e^{-2 \alpha s} d x^{\prime} d s .
\end{aligned}
$$

From the Sobolev embedding

$$
\left(\frac{n}{2}-\frac{n-1}{2 q}\right) \frac{1}{\mu} \leq 1 \Rightarrow W_{2}^{\mu}(\Omega) \hookrightarrow L_{2 q}(\partial \Omega)
$$

for $n=3, \mu=1, q=1$ we have

$$
\left\|D_{t, x^{\prime}}^{1} u_{\delta}\right\|_{2, \partial \Omega} \leq\left\|D_{t, x^{\prime}}^{1} u_{\delta}\right\|_{1,2, \Omega}, \quad\left\|u_{\delta}\right\|_{2, \partial \Omega} \leq\left\|u_{\delta}\right\|_{1,2, \Omega} .
$$

Using this and (4.41) we get

$$
\begin{aligned}
& \int_{\partial \Omega^{t}}\left|M D_{t, x^{\prime}}^{2} u_{\delta}-D_{t, x^{\prime}}^{2} M u_{\delta}\right|^{2} e^{-2 \alpha s} d x^{\prime} d s \\
\leq & c b^{2}\left[\left(a^{2 m}+1\right)\left(\left\|u_{\delta}\right\|_{1, \Omega^{t}, \alpha}^{2}+\left\|D_{t, x^{\prime}}^{2} u_{\delta}\right\|_{0, \Omega^{t}, \alpha}^{2}\right)+a^{2(m-1)}\left\|L u_{\delta}\right\|_{1, \Omega^{t}, \alpha}^{2}\right] .
\end{aligned}
$$

If we take $\alpha$ such that

$$
c\left[\frac{2}{\alpha \alpha_{0}} a^{2}\left(a^{2 m}+1\right)+\frac{\alpha \alpha_{0}}{2}\left(a^{4 m}+a^{2 m}\right)+c_{3} b^{2}\left(a^{2 m}+1\right)\right] \leq \frac{\alpha \alpha_{0}}{4}
$$

and use the inequality (following from (4.32))

$$
\begin{aligned}
\left\|D_{t, x^{\prime}}^{1} D_{x_{1}}^{1} u_{\delta}\right\|_{0,2, \Omega}^{2}+\left\|D_{x_{1}}^{2} u_{\delta}\right\|_{0,2, \Omega}^{2} & \\
\leq & c\left(a^{4(m-1)}+2 a^{2(m-1)}\right)\left\|L u_{\delta}\right\|_{1,0, \Omega}^{2} \\
& \quad+\left(2 a^{2 m}+a^{4 m}\right)\left\|u_{\delta}\right\|_{1,0, \Omega}^{2}+\left(a^{2 m}+a^{4 m}\right)\left\|D_{t, x^{\prime}}^{2} u_{\delta}\right\|_{0,2, \Omega}^{2}
\end{aligned}
$$

we conclude (combining (4.38), (4.40)-(4.42), (4.46) and using (4.43)-(4.45)) that

$$
\begin{aligned}
\alpha_{0}\left|u_{\delta}\right|_{2,0, \Omega}^{2} e^{-2 \alpha t}+\frac{\alpha \alpha_{0}}{4}\left\|u_{\delta}\right\|_{2, \Omega^{t}, \alpha}^{2}+\frac{c_{0}}{2}\left\|u_{\delta}\right\|_{2, \partial \Omega^{t}, \alpha}^{2} \\
\leq p_{2}(a, b)\left(\left\|L u_{\delta}\right\|_{2, \Omega^{t}, \alpha}^{2}+\left\|L u_{\delta}\right\|_{1,0, \Omega}^{2} e^{-2 \alpha t}\right) \\
\quad+q_{2}(a, b)\left\|M u_{\delta}\right\|_{2, \partial \Omega^{t}, \alpha}^{2}+\left.c_{2}\left|u_{\delta}\right|_{2,0, \Omega}^{2}\right|_{t=0}
\end{aligned}
$$

where $p_{2}, q_{2}$ are polynomials. 
Moreover, using

$$
|F|_{\nu, 0, \Omega}^{2} e^{-2 \alpha t} \leq \frac{c}{\alpha}|F|_{\nu+1, \Omega^{t}, \alpha}^{2}+\left.|F|_{\nu, 0, \Omega}^{2}\right|_{t=0}
$$

for $\nu=1$ and taking $\delta \rightarrow 0$, for $u=\lim _{\delta \rightarrow 0} u_{\delta}$ we obtain estimate (4.28) for $s=2$.

Finally, we consider $s=3$; like before, by (4.9) we get

$$
\begin{aligned}
\alpha_{0} e^{-2 \alpha t} \int_{\Omega}\left|D_{t, x^{\prime}}^{3} u_{\delta}\right|^{2} d x & +\frac{\alpha \alpha_{0}}{2} \int_{\Omega^{t}}\left|D_{t, x^{\prime}}^{3} u_{\delta}\right|^{2} e^{-2 \alpha s} d x d s \\
& +\frac{c_{0}}{2} \int_{\partial \Omega^{t}}\left|D_{t, x^{\prime}}^{3} u_{\delta}\right|^{2} e^{-2 \alpha s} d x^{\prime} d s \\
\leq & \frac{2}{\alpha \alpha_{0}} \int_{\Omega^{t}}\left|D_{t, x^{\prime}}^{3} L u_{\delta}-L D_{t, x^{\prime}}^{3} u_{\delta}\right|^{2} e^{-2 \alpha s} d x d s \\
& +\frac{2}{\alpha \alpha_{0}} \int_{\Omega^{t}}\left|D_{t, x^{\prime}}^{3} L u_{\delta}\right|^{2} e^{-2 \alpha s} d x d s \\
& +c_{3} \int_{\partial \Omega^{t}}\left|D_{t, x^{\prime}}^{3} M u_{\delta}-M D_{t, x^{\prime}}^{3} u_{\delta}\right|^{2} e^{-2 \alpha s} d x^{\prime} d s \\
& +c_{3} \int_{\partial \Omega^{t}}\left|D_{t, x^{\prime}}^{3} M u_{\delta}\right|^{2} e^{-2 \alpha s} d x^{\prime} d s \\
& +\left.c_{2} \int_{\Omega}\left|D_{t, x^{\prime}}^{3} u_{\delta}\right|^{2} d x\right|_{t=0}
\end{aligned}
$$

Because by (4.32),

$$
\begin{aligned}
& \int_{\Omega^{t}}\left|D_{t, x^{\prime}}^{2} D_{x_{1}}^{1} u_{\delta}\right|^{2} e^{-2 \alpha s} d x d s \\
& \quad \leq c a^{2(m-1)}\left[\left\|L u_{\delta}\right\|_{2, \Omega^{t}, \alpha}^{2}+a^{2}\left(\left\|u_{\delta}\right\|_{2, \Omega^{t}, \alpha}^{\prime 2}+\left\|D_{t, x^{\prime}}^{3} u_{\delta}\right\|_{0, \Omega^{t}, \alpha}^{2}\right)\right]
\end{aligned}
$$

we can estimate

$$
\begin{aligned}
\int_{\Omega^{t}} & \left|L D_{t, x^{\prime}}^{3} u_{\delta}-D_{t, x^{\prime}}^{3} L u_{\delta}\right|^{2} e^{-2 \alpha s} d x d s \\
\leq & \int_{\Omega^{t}} \mid D_{t, x^{\prime}}^{3} \widetilde{L} \cdot D_{t, x}^{1} u_{\delta} \\
& \quad+D_{t, x^{\prime}}^{2} \widetilde{L} \cdot D_{t, x^{\prime}}^{1} D_{t, x}^{1} u_{\delta}+\left.D_{t, x^{\prime}}^{1} \widetilde{L} \cdot D_{t, x^{\prime}}^{2} D_{t, x}^{1} u_{\delta}\right|^{2} e^{-2 \alpha s} d x d s \\
\leq & c a^{2}\left(\left\|u_{\delta}\right\|_{2, \Omega^{t}, \alpha}^{2}+\left\|D_{t, x^{\prime}}^{2} D_{t, x}^{1} u_{\delta}\right\|_{0, \Omega^{t}, \alpha}^{2}\right) \\
\leq & c a^{2 m}\left\|L u_{\delta}\right\|_{2, \Omega^{t}, \alpha}^{2}+a^{2}\left(a^{2 m}+1\right)\left(\left\|u_{\delta}\right\|_{2, \Omega^{t}, \alpha}^{2}+\left\|D_{t, x^{\prime}}^{3} u_{\delta}\right\|_{0, \Omega^{t}, \alpha}^{2}\right) .
\end{aligned}
$$


Let us estimate

$$
\begin{aligned}
& \int_{\Omega^{t}}\left|D_{t, x^{\prime}}^{1} D_{x_{1}}^{2} u_{\delta}\right|^{2} e^{-2 \alpha s} d x d s \\
& \leq c a^{2(m-1)}\left[\left\|L u_{\delta}\right\|_{2, \Omega^{t}, \alpha}^{2}+a^{2}\left(\left\|u_{\delta}\right\|_{2, \Omega^{t}, \alpha}^{2}+\left\|D_{t, x^{\prime}}^{2} D_{x_{1}}^{1} u_{\delta}\right\|_{0, \Omega^{t}, \alpha}^{2}\right)\right] \\
& \leq c\left[\left(a^{2(m-1)}+a^{4 m-2}\right)\left\|L u_{\delta}\right\|_{2, \Omega^{t}, \alpha}^{2}\right. \\
& \left.+\left(a^{2 m}+a^{4 m}\right)\left\|u_{\delta}\right\|_{2, \Omega^{t}, \alpha}^{2}+a^{4 m}\left\|D_{t, x^{\prime}}^{3} u_{\delta}\right\|_{0, \Omega^{t}, \alpha}^{2}\right], \\
& \text { (4.53) } \quad \int_{\Omega^{t}}\left|D_{x_{1}}^{3} u_{\delta}\right|^{2} e^{-2 \alpha s} d x d s \\
& \leq c a^{2(m-1)}\left[\left\|L u_{\delta}\right\|_{2, \Omega^{t}, \alpha}^{2}+a^{2}\left(\left\|u_{\delta}\right\|_{2, \Omega^{t}, \alpha}^{2}+\left\|D_{t, x}^{1} D_{x_{1}}^{2} u_{\delta}\right\|_{0, \Omega^{t}, \alpha}^{2}\right)\right] \\
& \leq c\left[\left(a^{2(m-1)}+a^{4 m-2}+a^{6 m-2}\right)\left\|L u_{\delta}\right\|_{2, \Omega^{t}, \alpha}^{2}\right. \\
& \left.+\left(a^{2 m}+a^{4 m}+a^{6 m}\right)\left\|u_{\delta}\right\|_{2, \Omega^{t}, \alpha}^{2}+a^{6 m}\left\|D_{t, x^{\prime}}^{3} u_{\delta}\right\|_{0, \Omega^{t}, \alpha}^{2}\right] .
\end{aligned}
$$

We have to consider

$$
\begin{aligned}
& \int_{\partial \Omega^{t}}\left|M D_{t, x^{\prime}}^{3} u_{\delta}-D_{t, x^{\prime}}^{3} M u_{\delta}\right|^{2} e^{-2 \alpha s} d x^{\prime} d s \\
& \quad=\int_{\partial \Omega^{t}}\left|\left(D_{t, x^{\prime}}^{3} M\right) u_{\delta}+D_{t, x^{\prime}}^{2} M \cdot D_{t, x^{\prime}}^{1} u_{\delta}+D_{t, x^{\prime}}^{1} M \cdot D_{t, x^{\prime}}^{2} u_{\delta}\right|^{2} e^{-2 \alpha s} d x^{\prime} d s \\
& \quad \leq c b^{2} \int_{0}^{t}\left(\left|u_{\delta}\right|_{2, \partial \Omega}^{2}+\left|D_{t, x^{\prime}}^{1} u_{\delta}\right|_{2, \partial \Omega}^{2}+\left|D_{t, x^{\prime}}^{2} u_{\delta}\right|_{2, \partial \Omega}^{2}\right) e^{-2 \alpha s} d s
\end{aligned}
$$

Using again the Sobolev embeddings

$$
\begin{aligned}
\left\|u_{\delta}\right\|_{2, \partial \Omega} & \leq c\left\|u_{\delta}\right\|_{1,2, \Omega}, \\
\left\|D_{t, x^{\prime}}^{1} u_{\delta}\right\|_{2, \partial \Omega} & \leq c\left\|D_{t, x^{\prime}}^{1} u_{\delta}\right\|_{1,2, \Omega}, \\
\left\|D_{t, x^{\prime}}^{2} u_{\delta}\right\|_{2, \partial \Omega} & \leq c\left\|D_{t, x^{\prime}}^{2} u_{\delta}\right\|_{1,2, \Omega},
\end{aligned}
$$

we obtain

$$
\begin{aligned}
& \int_{\partial \Omega^{t}}\left|M D_{t, x^{\prime}}^{3} u_{\delta}-D_{t, x^{\prime}}^{3} M u_{\delta}\right|^{2} e^{-2 \alpha s} d x^{\prime} d s \\
\leq & c b^{2}\left(\left\|u_{\delta}\right\|_{2, \Omega^{t}, \alpha}^{2}+\left\|D_{t, x^{\prime}}^{3} u_{\delta}\right\|_{0, \Omega^{t}, \alpha}^{2}+\left\|D_{x_{1}}^{1} D_{t, x^{\prime}}^{2} u_{\delta}\right\|_{0, \Omega^{t}, \alpha}^{2}\right) \\
\leq & c b^{2} a^{2(m-1)}\left\|L u_{\delta}\right\|_{2, \Omega^{t}, \alpha}^{2}+b^{2}\left(a^{2 m}+1\right)\left(\left\|u_{\delta}\right\|_{2, \Omega^{t}, \alpha}^{2}+\left\|D_{t, x^{\prime}}^{3} u_{\delta}\right\|_{0, \Omega^{t}, \alpha}^{2}\right) .
\end{aligned}
$$

Let us assume that

$$
c\left[\frac{2}{\alpha \alpha_{0}} a^{2}\left(a^{2 m}+1\right)+\frac{\alpha \alpha_{0}}{2}\left(a^{2 m}+a^{4 m}+a^{6 m}\right)+c_{3} b^{2}\left(a^{2 m}+1\right)\right] \leq \frac{\alpha \alpha_{0}}{4} .
$$


Then, adding to (4.49) inequalities (4.50), (4.52)-(4.53) and

$$
\begin{aligned}
\left\|D_{t, x^{\prime}}^{2} D_{x_{1}}^{1} u_{\delta}\right\|_{2, \Omega}^{2}+ & \left\|D_{t, x^{\prime}}^{1} D_{x_{1}}^{2} u_{\delta}\right\|_{2, \Omega}^{2}+\left\|D_{x_{1}}^{3} u_{\delta}\right\|_{2, \Omega}^{2} \\
\leq & c\left[\left(3 a^{2(m-1)}+2 a^{4 m-2}+a^{6 m-2}\right)\left\|L u_{\delta}\right\|_{2,0, \Omega}^{2}\right. \\
& +\left(3 a^{2 m}+2 a^{4 m}+a^{6 m}\right)\left\|u_{\delta}\right\|_{2,0, \Omega}^{2} \\
& \left.+\left(a^{2 m}+a^{4 m}+a^{6 m}\right)\left\|D_{t, x^{\prime}}^{3} u_{\delta}\right\|_{2, \Omega}^{2}\right]
\end{aligned}
$$

and using estimates (4.51), (4.52), by inequality (4.47), and the energy inequality (4.48) for $\nu=2$, we finally obtain

$$
\begin{aligned}
\alpha_{0}\left|u_{\delta}\right|_{3,0, \Omega}^{2} e^{-2 \alpha t}+ & \frac{\alpha \alpha_{0}}{4}\left\|u_{\delta}\right\|_{3, \Omega^{t}, \alpha}^{2}+\frac{c_{0}}{2}\left\|u_{\delta}\right\|_{3, \partial \Omega^{t}, \alpha}^{2} \\
\leq & p_{3}(a, b)\left(\left\|L u_{\delta}\right\|_{3, \Omega^{t}, \alpha}^{2}+\left.\left\|L u_{\delta}\right\|_{2,0, \Omega}^{2}\right|_{t=0}\right) \\
& \quad+\left.c_{2}\left\|u_{\delta}\right\|_{3,0, \Omega}^{2}\right|_{t=0}+q_{3}(a, b)\left\|M u_{\delta}\right\|_{3, \partial \Omega^{t}, \alpha}^{2}
\end{aligned}
$$

where $p_{3}, q_{3}$ are polynomials.

Moreover, by convergence in suitable spaces, after passing with $\delta$ to zero, we obtain estimate (4.28) for $s=3$. This concludes the proof.

Theorem 4.2 and Lemma 4.5 imply:

THEOREM 4.3. Suppose the following assumptions are satisfied:

(1) $\Omega$ is a half-space, $\widetilde{L} \in \Pi_{0}^{3}\left(\Omega^{T}\right), M \in \Pi_{0}^{3}\left(\Omega^{T}\right) \cap H_{\alpha}^{3}\left(\partial \Omega^{t}\right), F \in$ $H_{\alpha}^{3}\left(\Omega^{T}\right), g \in H_{\alpha}^{3}\left(\partial \Omega^{T}\right), u_{0} \in H^{3}(\Omega)$ and $\left.u_{0}\right|_{\partial \Omega}=0$.

(2) We have

$$
\begin{aligned}
& \min _{\mu} \min _{\Omega^{T}}\left|\lambda_{\mu}\right| \geq c_{0}>0 \quad \text { so } \quad\left|\operatorname{det} A_{\bar{n}}\right| \geq c_{0}^{m}, \text { and } \\
& \max _{\Omega^{T}}\left|\lambda_{\mu}\right| \leq \frac{\max _{\Omega^{T}}\left|\operatorname{det} A_{\bar{n}}\right|}{c_{0}^{m-1}} \leq c \frac{1}{c_{0}^{m-1}}|\widetilde{L}|_{3,0, \infty, \Omega^{T}}^{m} .
\end{aligned}
$$

(3) $E u \cdot u \geq \alpha_{0} u^{2}$.

Then there exists a unique solution of problem (4.1) such that $u \in \Pi_{0}^{3}\left(\Omega^{T}\right) \cap$ $H_{\alpha}^{3}\left(\Omega^{T}\right) \cap H_{\alpha}^{3}\left(\partial \Omega^{T}\right)$ and estimate (4.28) holds under assumption (4.29).

4(c) Existence of solutions for the linearized equations in a bounded domain. Now we want to prove Theorem 4.3 for a bounded domain $\Omega$. Since (4.9) holds in $\Omega$, we have the existence of solutions to the linearized problem (4.1) in $L_{2, \alpha}\left(\Omega^{T}\right)$ (by Lemma 4.1 and Theorem 4.1). For higher regularity we introduce a suitable partition of unity.

Take a system of $\xi_{i}(x) \in C^{\infty}(\Omega), \xi_{i} \in[0,1], i \in \mathcal{M} \cup \mathcal{N}, \Omega_{i}=\operatorname{supp} \xi_{i}(x)$ $\cap \Omega, w_{i}=\left\{x: \xi_{i}(x)=1\right\}$,

$$
i \in \mathcal{M} \Leftrightarrow \Omega_{i} \cap \partial \Omega=\emptyset, \quad i \in \mathcal{N} \Leftrightarrow \Omega_{i} \cap \partial \Omega \neq \emptyset,
$$


$\bigcup \Omega_{i}=\bigcup w_{i}=\Omega$, $\operatorname{diam} \Omega_{i} \leq \lambda$, only finitely many $\Omega_{i}$ 's are nonempty. Next, let $\eta_{i}(x)=\xi_{i}(x) / \sum \xi_{i}^{2}(x)$ (so $\left.\sum \eta_{i}(x) \xi_{i}(x)=1\right)$. We define $f_{i}(x, t)=$ $f(x, t) \eta_{i}(x)$; from (4.1) we get

$$
\begin{aligned}
L u_{i} & =L\left(u \eta_{i}\right)=E\left(u \eta_{i}\right)_{t}+\sum_{\gamma=1}^{3} A_{\gamma}\left(u \eta_{i}\right)_{x_{\gamma}} \\
& =E \frac{\partial}{\partial t} u \cdot \eta_{i}+\sum_{\gamma=1}^{3}\left(A_{\gamma} \frac{\partial}{\partial x_{\gamma}} u \cdot \eta_{i}+A_{\gamma} u \cdot \frac{\partial}{\partial x_{\gamma}} \eta_{i}\right) \\
& =(L u) \eta_{i}+\sum_{\gamma=1}^{3} A_{\gamma} u \cdot \eta_{i, x_{\gamma}}
\end{aligned}
$$

so

$$
\begin{aligned}
& L u_{i}=F_{i}+\left[L, \eta_{i}\right] u \quad \text { in } \Omega_{i}, \\
& \left.M u_{i}\right|_{\partial \Omega}=g_{i}, \\
& \left.u_{i}\right|_{t=0}=u_{0 i}, \quad \text { where }\left[L, \eta_{i}\right] u=\sum_{\alpha=1}^{3} A_{\alpha} u \eta_{i, x_{\alpha}} .
\end{aligned}
$$

We consider two cases. For $i \in \mathcal{M}$ we have only the Cauchy problem $(4.58)_{1,3}$, so we obtain an estimate of type (4.28), but without the boundary term and expressions with $M$; denote it by $(4.28)^{\prime}$. In the case $i \in \mathcal{N}$, we take a local coordinate system centred in the middle of $\partial \Omega \cap \Omega_{i}$ such that $x_{1}>0$ belong to $\Omega_{i}$ and $x_{1}$ is a coordinate along the axis generated by $\bar{n}\left(\widetilde{x}_{i}\right)$ and $x^{\prime}=\left(x_{2}, x_{3}\right)$ are directions perpendicular to $\bar{n}$. Then, if $\partial \Omega \cap \Omega_{i}$ is described by $x_{1}-\varphi\left(x^{\prime}\right)=0$, by the transformation $y^{\prime}=x^{\prime}, y_{1}=x_{1}-\varphi\left(x^{\prime}\right)$ we get the half-space $y_{1}>0$. We can write our problem in the form

$$
\begin{aligned}
& \widehat{L} \widehat{u}_{i}=\widehat{F}_{i}+\left[\widehat{L}, \widehat{\eta}_{i}\right] u, \\
& \left.\widehat{M} \widehat{u}_{i}\right|_{\partial \Omega}=\widehat{g}_{i}, \\
& \left.\widehat{u}_{i}\right|_{t=0}=\widehat{u}_{0 i}, \text { where } \widehat{f}(y)=\left.f(x)\right|_{x=x(y)},
\end{aligned}
$$

and $\bar{n}=\left(-1, \varphi_{x^{\prime}}\right)\left(1+\varphi_{x^{\prime}}^{2}\right)^{-1 / 2}=-\left(\frac{\partial y_{1}}{\partial x_{1}}, \frac{\partial y_{1}}{\partial x_{2}}, \frac{\partial y_{1}}{\partial x_{3}}\right)\left(\sum_{i=1}^{3} y_{1, x_{i}}^{2}\right)^{-1 / 2}$ implies

$$
\sum_{s=1}^{3} \widehat{A}_{s}\left(-\frac{\partial y_{1}}{\partial x_{s}}\right)\left(\sum_{i=1}^{3} y_{1, x_{i}}^{2}\right)^{-1 / 2}=\widehat{A} \cdot \bar{n}
$$

so we have new matrices $A_{1}^{\prime}=-\widehat{A} \cdot \bar{n}, A_{2}^{\prime}=\widehat{A}_{2}, A_{3}^{\prime}=\widehat{A}_{3}$ (symmetric).

We can apply the considerations of part (b) to obtain an estimate for system (4.59) of type (4.28). Notice that in both cases $i \in \mathcal{M}$ and $i \in \mathcal{N}$ we must additionally consider the second term on the right-hand side of $(4.58)_{1}$ and $(4.59)_{1}$, respectively. We can write 


$$
\begin{aligned}
\left\|\left[L, \eta_{i}\right] u\right\|_{\mu, \Omega^{t}, \alpha} & =\left\|\sum_{k=1}^{3} A_{k} u \eta_{i, x_{k}}\right\|_{\mu, \Omega^{t}, \alpha} \\
& =\left\|\sum_{k=1}^{3} A_{k} u_{i} \xi_{i}(x) \eta_{i, x_{k}}(x)\right\|_{\mu, \Omega^{t}, \alpha} \\
& \leq c\left\|\sum_{k=1}^{3} A_{k} u_{i}\right\|_{3, \Omega^{t}, \alpha} \leq c a\left\|u_{i}\right\|_{3, \Omega^{t}, \alpha}
\end{aligned}
$$

because $\xi_{i} \leq 1, \eta_{i} \in C^{\infty}(\Omega)$.

We obtain, for (4.59) and $i \in \mathcal{N}$,

$$
\begin{aligned}
\alpha_{0}\left|\widehat{u}_{i}\right|_{\mu, 0, \widehat{\Omega}_{i}}^{2} e^{-2 \alpha t}+ & \frac{\alpha \alpha_{0}}{4}\left\|\widehat{u}_{i}\right\|_{\mu, \widehat{\Omega}_{i}^{t}, \alpha}^{2} e^{-2 \alpha t} \\
& +\frac{\alpha \alpha_{0}}{4}\left\|\widehat{u}_{i}\right\|_{\mu, \widehat{\Omega}_{i}^{t}, \alpha}^{2}+\frac{c_{0}}{2}\left\|\widehat{u}_{i}\right\|_{\mu, \partial \widehat{\Omega}_{i}^{t}, \alpha}^{2} \\
\leq & \bar{p}_{\mu}(a, b)\left[\left\|\widehat{F}_{i}\right\|_{\mu, \widehat{\Omega}_{i}^{t}, \alpha}^{2}+\left.\left|\widehat{F}_{i}\right|_{\mu-1,0, \widehat{\Omega}_{i}}^{2}\right|_{t=0}\right] \\
& \quad+\bar{q}_{\mu}(a, b)\left\|\widehat{g}_{i}\right\|_{\mu, \partial \widehat{\Omega}_{i}^{t}, \alpha}^{2}+\left.\bar{r}_{\mu}(a, b)\left|\widehat{u}_{i}\right|_{\mu, 0, \widehat{\Omega}_{i}}^{2}\right|_{t=0} .
\end{aligned}
$$

where

$$
\bar{p}_{\mu}(a, b)+c a^{2} \leq \alpha \alpha_{0},
$$

$\bar{p}_{\mu}, \bar{r}_{\mu}, \bar{q}_{\mu}, \bar{p}_{0}$ are polynomials, $\widehat{\Omega}_{i}=T \Omega_{i}, \widehat{F}_{i}=(\widehat{L} u)_{i}, \widehat{g}_{i}=(\widehat{M} u)_{i}$ and $T$ is the transformation defined by $y=y(x)$.

By summing inequalities (4.61) over $i \in \mathcal{N}$ and (4.28)' over $i \in \mathcal{M}$, using $u=\sum_{i \in \mathcal{M} \cup \mathcal{N}} u_{i}(x) \xi_{i}(x)$, we obtain an estimate of the form (4.28) for a bounded domain $\Omega$. Assuming

$$
\alpha \alpha_{0} \geq p_{0}(a, b)+c a^{2}
$$

for a bounded domain, we formulate:

THEOREM 4.4. Let $\Omega$ be a bounded domain with $\partial \Omega \in C^{3}$. Let the assumptions of Theorem 4.3 and (4.63) be satisfied for $\Omega$. Then there exists a unique solution $w$ of the linearized problem (3.1), where $u \in \Pi_{0}^{3}\left(\Omega^{t}\right) \cap$ $H_{\alpha}^{3}\left(\Omega^{t}\right) \cap H_{\alpha}^{3}\left(\Omega^{t}\right) \cap H_{\alpha}^{3}\left(\partial \Omega^{t}\right)$ and (4.28) holds.

5. The existence and uniqueness of solution of problem (3.1). We will consider the following iteration scheme:

$$
\begin{aligned}
& L\left(u_{m}\right) u_{m+1} \equiv E\left(t, x, u_{m}\right) u_{m+1, t}+\sum_{i=1}^{3} A_{i}\left(x, t, u_{m}\right) u_{m+1, x_{i}}=0, \\
& M\left(t, x, u_{m}\right) u_{m+1}=g(t, x) \quad \text { on } \partial \Omega^{t}, \\
& \left.u_{m+1}\right|_{t=0}=u_{0}(x) \quad \text { in } \Omega,
\end{aligned}
$$

for $m=0,1,2, \ldots$. 
Define $Q\left(G_{0}, \delta\right)=\left\{u: \Omega^{t} \rightarrow \mathbb{R}^{m}: \sup _{\Omega^{t}}\left|u(x, s)-u_{0}(x)\right| \leq \delta\right.$ for some $u_{0}$ with values in $\left.G_{0}\right\}$. Recall that we assume that $\bar{G} \subset G$. If the values of all functions in $Q\left(G_{0}, \delta\right.$ ) lie in $G$ (where $\delta$ depends on $t$ and the system of equations), we assume for $u \in Q\left(G_{0}, \delta\right)$ :

(a) The matrices $E, A_{i}$ are symmetric, $E$ is uniformly positive definite, and the matrix $-A_{\bar{n}}=-A \cdot \bar{n}$, where $\bar{n}$ is the unit outward vector normal to the boundary $\partial \Omega$, has eigenvalues separated from zero and positive eigenvalues bounded in a neighbourhood of the boundary.

(b) The matrix $M$ has the following form:

$$
\begin{aligned}
M= & \sum_{\mu, \nu=1}^{k} \alpha_{\mu \nu}\left(t, x^{\prime}, u\right) \gamma_{\mu}^{+}\left(t, x^{\prime}, u\right) \gamma_{\nu}^{+}\left(t, x^{\prime}, u\right) \\
& +\sum_{\mu=1}^{k} \sum_{\nu=k+1}^{m} \beta_{\mu \nu}\left(t, x^{\prime}, u\right) \gamma_{\mu}^{+}\left(t, x^{\prime}, u\right) \gamma_{\nu}^{-}\left(t, x^{\prime}, u\right)
\end{aligned}
$$

and

$$
\max _{\partial \Omega^{t}}\left|\alpha_{\mu \nu}^{-1}\right| \leq \delta_{0}^{-1}, \quad \max _{\partial \Omega^{t}}\left|\beta_{\mu \nu}\right| \leq \beta_{0} \quad \forall \mu, \nu .
$$

(c) $\operatorname{det} A_{\bar{n}}(t, x, u(x, t)) \neq 0$ in a neighbourhood of the boundary.

(d) The matrices $E(x, t, u(x, t)), A_{i}(x, t, u(x, t))$ are 3 -times differentiable functions with respect to $t, x, u$, and belong to $L_{2}(\Omega)$ for each $t$.

We can guarantee that conditions (a)-(d) are satisfied for $u \in Q\left(G_{0}, \delta\right)$ in the following way. By Theorem 4.4 every solution of system (5.1) belongs to $C^{\beta}\left(\Omega^{t}\right), \beta \in(0,1)$. Using continuity of $u$ with respect to $t$, condition (d) and the assumption that conditions (a)-(d) are satisfied for $u_{0}(x)=\left.u\right|_{t=0} \in G_{0}$ we have these properties for $u \in Q\left(G_{0}, \delta\right)$ for sufficiently small $t$; so let $t^{*}$ be a time such that for $t<t^{*}$ we can use Theorem 4.4 for each $u_{m}$.

Let us assume $\left.u_{0}\right|_{\partial \Omega}=0$ and consider, for $v_{m}=u_{m}-u_{0}$, the following system:

$$
\begin{aligned}
& L v_{m+1}=E\left(t, x, u_{m}\right) v_{m+1, t}+\sum_{i=1}^{3} A_{i}\left(t, x, u_{m}\right) v_{m+1, x_{i}} \\
& =-\sum_{i=1}^{3} A_{i}\left(t, x, u_{m}\right) u_{0, x_{i}}, \\
& \left.M v_{m+1}\right|_{\partial \Omega}=g, \\
& \left.v_{m+1}\right|_{t=0}=0 .
\end{aligned}
$$

We get, by Theorem 4.4 ,

$$
\begin{aligned}
\left\|\left|v_{m+1}\right|\right\|_{3}^{2} \leq & \widehat{p}\left(Q,\left\||| u_{m} \mid\right\|_{3}\right)\left[\left\|A u_{0, x}\right\|_{3, \Omega^{t}, \alpha}^{2}+\left.\left|A u_{0, x}\right|_{2,0, \Omega}^{2}\right|_{t=0}\right] \\
& +\widehat{q}\left(Q,\left\|\left|\left\|u_{m}\right\|\right|_{3}\right)\|g\|_{3, \partial \Omega^{t}, \alpha}^{2}\right. \\
& +\left.\widehat{p}_{0}\left(Q,\left.\left\|u_{m}\right\|\right|_{3}\right)\left|v_{m+1}\right|_{3,0, \Omega}^{2}\right|_{t=0}
\end{aligned}
$$


where $\left|\left\|v\left|\left\|_{3}^{2} \equiv|v|_{3,0, \infty, \Omega^{t}}^{2}+\right\| v \|_{3, \Omega^{t}, \alpha}^{2}, \widehat{p}_{0}\left(Q,\left|\left\|u_{m} \mid\right\|_{3}\right) \geq\left|E\left(t, x, u_{m}\right)\right|\right.\right.\right.\right.$, $\widehat{p}\left(Q,\left|\|u \mid\|_{3}\right)>p(a, b), \widehat{q}\left(Q, \mid\|u\|_{3}\right)>q(a, b)\right.$ and $a, b$ are defined as before. By the definition of $v$, we have

$$
\left\||| u\left|\left\|_{3} \leq\right\| v\right|\right\|_{3}+\left\|u_{0}\right\|_{3,2, \Omega} .
$$

To prove convergence of $\left\{u_{m}\right\}$ we have to know that if $\left\|v_{m}\right\|_{3} \leq d$ then $\left\|v_{m+1}\right\| \|_{3} \leq d^{2}$; using (5.4) in (5.3) we have

$$
\begin{aligned}
\left.\left\|v_{m+1}\right\|\right|_{3} ^{2} \leq & \widehat{p}\left(Q, d+\left|\left\|u_{0}|\||_{3,2, \Omega}\right)\left[\left\|A u_{0, x}\right\|_{3, \Omega^{t}, \alpha}^{2}+\left.\left|A u_{0, x}\right|_{2,0, \Omega}^{2}\right|_{t=0}\right]\right.\right. \\
& +\widehat{q}\left(Q, d+\left\|u_{0}\right\|_{3,2, \Omega}\right)\|g\|_{3, \partial \Omega^{t}, \alpha}^{2} \\
& +\left.\widehat{p}_{0}\left(Q, d+\left\|u_{0}\right\|_{3,2, \Omega}\right)\left|v_{m+1}\right|_{3,0, \Omega}^{2}\right|_{t=0} .
\end{aligned}
$$

REMARK. We have used $\widehat{p}\left(Q, \mid\|u\|_{3}\right)$ and $\widehat{q}\left(Q,\||u|\|_{3}\right)$ by Lemma 6.1 of $[10]$.

We see that $\| v_{m+1}||_{3}^{2} \leq d^{2}$ for sufficiently small norms of $u_{0, x}$ and $g$, where $\|g\|_{3, \partial \Omega^{t}, \alpha}$ depends on the time $t^{*}$. This guarantees the convergence of the sequence $\left\{u_{m}\right\}$. Introducing $U_{m}=u_{m}-u_{m-1}=v_{m}-v_{m-1}$ we have the problem

$$
\begin{aligned}
& L\left(u_{m}\right) U_{m+1}=-\left[L\left(u_{m}\right)-L\left(u_{m-1}\right)\right] v_{m} \\
&-\sum_{i=1}^{3}\left[A_{i}\left(u_{m}\right)-A_{i}\left(u_{m-1}\right)\right] u_{0, x_{i}}, \\
& M\left(u_{m}\right) U_{m+1}=-\left[M\left(u_{m}\right)-M\left(u_{m-1}\right)\right] v_{m}, \\
&\left.U_{m+1}\right|_{t=0}=0, \quad m \geq 0, \quad U_{0}=u_{0}(x) .
\end{aligned}
$$

By (d) we can write

$$
\begin{aligned}
\| \widetilde{L}\left(u_{m}\right)-\widetilde{L}\left(u_{m-1}\right)||_{3} & \leq\left.|\widetilde{L}|_{3,0, \infty, \Omega^{T}}||\left|u_{m}-u_{m-1}\right|\right|_{3}, \\
||\left|M\left(u_{m}\right)-M\left(u_{m-1}\right)\right| \|_{3} & \leq\left.|M|_{3,0, \infty, \Omega^{T}}||\left|u_{m}-u_{m-1}\right|\right|_{3},
\end{aligned}
$$

therefore by Theorem 4.4 for problem (5.6) we have

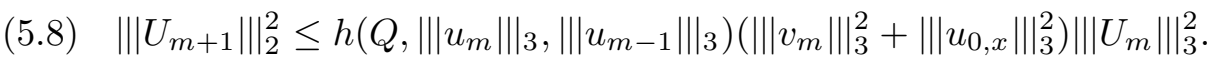

By the smallness of $\left\|\left|v_{m}\right|\right\|_{3}$, adding the assumption that $\left\|u_{0, x}\right\|_{3,2, \Omega}$ is sufficiently small, we have the convergence of $\left\{u_{m}\right\}$ to $u$ in $L_{\infty}\left(0, t ; L_{2}(\Omega)\right)$ $\cap L_{2, \alpha}\left(\Omega^{t}\right) \cap L_{2, \alpha}\left(\partial \Omega^{t}\right)$ and by (5.4), (5.5),

$$
u \in \Pi_{0}^{3}\left(\Omega^{t}\right) \cap H_{\alpha}^{3}\left(\Omega^{t}\right) \cap H_{\alpha}^{3}\left(\partial \Omega^{t}\right) .
$$

Moreover, $u$ is a unique solution. Assume, on the contrary, that $u_{1}, u_{2}$ are two solutions of the problem and $U=u_{1}-u_{2}$. Then

$$
\begin{aligned}
& L\left(u_{2}\right) U=-\left[L\left(u_{1}\right)-L\left(u_{2}\right)\right] u_{1}, \\
& M\left(u_{2}\right) U=-\left[M\left(u_{1}\right)-M\left(u_{2}\right)\right] u_{1}, \\
& \left.U\right|_{t=0}=0 .
\end{aligned}
$$


Lemma 4.1 implies

$$
\begin{aligned}
\alpha_{0}\|U\|_{2, \Omega}^{2} e^{-2 \alpha t}+\frac{\alpha \alpha_{0}}{2} & \|U\|_{0, \Omega^{t}, \alpha}^{2}+\frac{c_{0}}{2}\|U\|_{0, \partial \Omega^{t}, \alpha}^{2} \\
\leq & \left.\frac{2}{\alpha \alpha_{0}}\left\|\left[L\left(u_{1}\right)-L\left(u_{2}\right)\right] u_{1}\right\|\right|_{0, \Omega^{t}, \alpha} ^{2} \\
& \quad+\left(c_{0}+c_{1}\right) \delta_{0}^{-2}\left\|\left[M\left(u_{1}\right)-M\left(u_{2}\right)\right] u_{1}\right\|_{0, \partial \Omega^{t}, \alpha} .
\end{aligned}
$$

We can estimate

$$
\begin{gathered}
\left\|\left[L\left(u_{1}\right)-L\left(u_{2}\right)\right] u_{1}\right\|_{0, \Omega^{t}, \alpha} \leq \sup _{G} \sup _{\Omega^{t}}\left|\bar{L}^{\prime}(\widetilde{u})\right| \sup _{\Omega^{t}}\left(\left|u_{1}\right|+\left|D_{t, x}^{1} u_{1}\right|\right)\|U\|_{0, \Omega^{t}, \alpha}, \\
\left\|\left[M\left(u_{1}\right)-M\left(u_{2}\right)\right] u_{1}\right\|_{0, \partial \Omega^{t}, \alpha} \leq \sup _{G} \sup _{\partial \Omega^{t}}\left|M^{\prime}(\widetilde{u})\right| \sup _{\Omega^{t}}\left|u_{1}\right| \cdot\|U\|_{0, \partial \Omega^{t}, \alpha} .
\end{gathered}
$$

It is enough to assume that

$$
\begin{gathered}
\sup _{G} \sup _{\Omega^{t}}\left|\widetilde{L}^{\prime}(\widetilde{u})\right| \sup _{\Omega^{t}}\left(\left|u_{1}\right|+\left|D_{t, x}^{1} u_{1}\right|\right) \leq\left(\alpha \alpha_{0}\right)^{2} / 8, \\
\quad\left(c_{0}+c_{1}\right) \delta_{0}^{-2} \sup _{G} \sup _{\partial \Omega^{t}}\left|M^{\prime}(\widetilde{u})\right| \sup _{\Omega^{t}}\left|u_{1}\right| \leq c_{0} / 4
\end{gathered}
$$

to obtain

$$
\alpha_{0}\|U\|_{2, \Omega}^{2} e^{-2 \alpha t}+\frac{\alpha \alpha_{0}}{4}\|U\|_{0, \Omega^{t}, \alpha}^{2}+\frac{c_{0}}{4}\|U\|_{0, \partial \Omega^{t}, \alpha}^{2} \leq 0
$$

and this implies uniqueness.

Thus, we have proved:

TheOrem 5.1. Suppose the following assumptions are satisfied:

(1) $g \in H_{\alpha}^{3}\left(\partial \Omega^{t}\right),\left.u_{0}\right|_{\partial \Omega}=0, u_{0} \in H^{4}(\Omega)$.

(2) $\partial \Omega \in C^{3}$.

(3) The assumptions (a)-(d) are satisfied, and $\left\|u_{0, x}\right\|_{3,2, \Omega}$ is sufficiently small.

(4) $t \leq t^{*}$

(5) $\alpha$ satisfies $\alpha \alpha_{0} \geq \widehat{p}_{0}\left(Q, d+\left\|u_{0}\right\|_{3,2, \Omega}\right), \widehat{p}_{0}\left(Q, d+\left\|u_{0}\right\|_{3,2, \Omega}\right) \geq p_{0}(a, b)$ $+c a^{2}$, where $p_{0}, \widehat{p}_{0}$ are polynomials.

(6) (5.9), (5.10) are satisfied for some solution $u_{1} \in C^{1}\left(\Omega^{t}\right)$.

Then there exists a unique solution $u$ of (3.1) such that $u \in \Pi_{0}^{3}\left(\Omega^{t}\right) \cap$ $H_{\alpha}^{3}\left(\Omega^{t}\right) \cap H_{\alpha}^{3}\left(\partial \Omega^{t}\right)$ and we have uniqueness in $C^{1}\left(\bar{\Omega}^{t}\right)$.

6. Equations of relativistic hydrodynamics - existence and uniqueness of solutions for the mixed problem. We have proved existence and uniqueness of solutions for the initial-boundary problem (3.1) using assumptions (a)-(d) (see Section 5). To apply these results to problem (2.2) (that is, the symmetric system of relativistic hydrodynamics), we have to check the assumptions of Theorem 5.1. 
We have

$$
\begin{aligned}
& A^{0} z \cdot z=\left(\begin{array}{l}
\frac{p}{\beta} T_{p} s_{p}+\beta u_{1}^{2}+\beta u_{2}^{2}+\beta u_{3}^{2}+\frac{\delta}{\beta} s_{p} T_{\delta} \\
p \beta u_{1}-\beta u_{1}^{3} w+\frac{w}{\beta} u_{1}-\beta u_{1} u_{2}^{2} w-\beta u_{1} u_{3}^{2} w \\
p \beta u_{2}-\beta u_{1}^{2} u_{2} w-\beta u_{2}^{3} w+\frac{w}{\beta} u_{2}-\beta u_{2} u_{3}^{2} w \\
p \beta u_{3}-\beta u_{3} u_{1}^{2} w-\beta u_{3} u_{2}^{2} w-\beta u_{3}^{3} w+\frac{w}{\beta} u_{3} \\
\frac{p}{\beta} s_{p} T_{\delta}+\frac{\delta}{\beta} T_{\delta}\left(s_{\delta}-s / \delta\right)
\end{array}\right)^{T}\left(\begin{array}{c}
p \\
u^{1} \\
u^{2} \\
u^{3} \\
\delta
\end{array}\right) \\
& =p^{2} \frac{s_{p} T_{p}}{\beta}+2 p \delta \frac{s_{p} T_{\delta}}{\beta}+\delta^{2} \frac{T_{\delta}}{\beta}\left(s_{\delta}-s / \delta\right) \\
& +\left(u_{1}^{2}+u_{2}^{2}+u_{3}^{2}\right)(2 p \beta+w / \beta) \\
& -\left(u_{1}^{2}+u_{2}^{2}+u_{3}^{2}\right) \beta w
\end{aligned}
$$

so from $u_{1}^{2}+u_{2}^{2}+u_{3}^{2}=1 / \beta^{2}-1$ we get

$$
\begin{aligned}
A^{0} z \cdot z= & p^{2} \frac{s_{p} T_{p}}{\beta}+2 p \delta \frac{s_{p} T_{\delta}}{\beta}+\delta^{2} \frac{T_{\delta}\left(s_{\delta}-s / \delta\right)}{\beta} \\
& +\left(u_{1}^{2}+u_{2}^{2}+u_{3}^{2}\right)(2 p \beta+w \beta) .
\end{aligned}
$$

Lemma 6.1. Assume that there exists a constant $\varrho \in(0,1)$ such that for the initial data $z_{0}=\left(p_{0}, u_{01}, u_{02}, u_{03}, \delta_{0}\right), u_{\alpha}=v_{\alpha} /(c \beta)$ ( $v_{\alpha}$ is the velocity) we have

$$
v_{0}^{2} \leq\left(1-\varrho^{2}\right) c^{2}, \quad p_{0}>0, \quad \delta_{0}>0,
$$

and, for some $\varepsilon>0$,

$$
\begin{aligned}
& \delta_{0}>c_{1}\left(p_{0}+\varepsilon\right)+\varepsilon, \\
& \delta_{0}<c_{1}^{-1}\left(p_{0}-\varepsilon\right)\left(\gamma+\log \left\{\frac{p_{0}-\varepsilon}{\gamma-1}\left(\delta_{0}+\varepsilon\right)^{\gamma}\right\}\right)-\varepsilon
\end{aligned}
$$

where $c_{1}=2 / \varrho^{2}-1+6 \varepsilon^{2}$ and $\gamma$ is the adiabatic exponent. Then for $z \in$ $Q\left(G_{0}, \varepsilon\right)$ there exists $\alpha_{0}>0$ such that $E z \cdot z \geq \alpha_{0} z^{2}$ where $E=A^{0}$.

Pr o of. By definition of $Q\left(G_{0}, \varepsilon\right),\left|z(t)-z_{0}\right| \leq \varepsilon$, so $p>p_{0}-\varepsilon, \delta \geq \delta_{0}-\varepsilon$, $u_{\alpha} \leq u_{0 \alpha}+\varepsilon$. (6.2) implies

$$
\left.\beta\right|_{t=0}=\sqrt{1-v_{0}^{2} / c^{2}} \geq \varrho^{2}
$$

so we have

$$
\begin{aligned}
1 / \beta^{2}-1 & =\sum_{\alpha=1}^{3} u_{\alpha}^{2} \leq \sum_{\alpha=1}^{3}\left(u_{0 \alpha}+\varepsilon\right)^{2} \leq 2 \sum_{\alpha=1}^{3} u_{0 \alpha}^{2}+6 \varepsilon^{2} \\
& =2\left(1 /\left.\beta^{2}\right|_{t=0}-1\right)+6 \varepsilon^{2} \leq 2 / \varrho^{2}-2+6 \varepsilon^{2}
\end{aligned}
$$

hence

$$
\beta \geq\left(2 / \varrho^{2}-1+6 \varepsilon^{2}\right)^{-1} .
$$


From the state equation $p / \delta=R T$ we calculate

$$
T_{p}=\frac{1}{R} \cdot \frac{1}{\delta}, \quad T_{\delta}=-\frac{1}{R} \cdot \frac{p}{\delta^{2}} .
$$

Taking entropy in the form $s-s_{0}=c_{v} \log \left\{p /\left((\gamma-1) \delta^{\gamma}\right)\right\}$ where $c_{v}$ is the specific heat at constant volume, we get

$$
s_{p}=c_{v} / p, \quad s_{\delta}=-\gamma c_{v} / \delta .
$$

Assuming $\varepsilon<\min \left\{p_{0}, \delta_{0}\right\}$ and using (i), (ii) and (6.4), by the inequality $2 p \delta<p^{2}+\delta^{2}$, we can estimate

$$
\begin{aligned}
E z \cdot z \geq & p^{2} s_{p} T_{p}-\left(p^{2}+\delta^{2}\right) s_{p}\left|T_{\delta}\right| c_{1}+\delta^{2}\left(s_{\delta}-s / \delta\right) T_{\delta} \\
& +3\left(p_{0}-\varepsilon\right)\left(2 / \varrho^{2}-1+6 \varepsilon^{2}\right)^{-1} u^{2} \\
= & p^{2} s_{p}\left(T_{p}-c_{1}\left|T_{\delta}\right|\right)+\delta^{2}\left|T_{\delta}\right|\left(\left|s_{\delta}\right|+s / \delta-c_{1} s_{p}\right) \\
& +3\left(p_{0}-\varepsilon\right)\left(2 / \varrho^{2}-1+6 \varepsilon^{2}\right)^{-1} u^{2} .
\end{aligned}
$$

Assumptions (6.3) guarantee that for $z \in Q\left(G_{0}, \varepsilon\right), T_{p}-c_{1}\left|T_{\delta}\right|>0$ and $\left|s_{\delta}\right|+s / \delta-c_{1} s_{p}>0$, so we can estimate $E z \cdot z \geq \alpha_{0} z^{2}$, where

$$
\alpha_{0}=\min \left\{3 c_{1}^{-1}\left(p_{0}-\varepsilon\right), s_{p}\left(T_{p}-c_{1}\left|T_{\delta}\right|\right),\left|T_{\delta}\right|\left(\left|s_{\delta}\right|+s / \delta-c_{1} s_{p}\right)\right\},
$$

which concludes the proof.

REMARK 6.1. To satisfy (6.3) we need

$$
\left(\frac{1+v_{0}^{2} / c^{2}}{1-v_{0}^{2} / c^{2}}\right)^{2}<\log \frac{e^{\gamma} R T_{0}}{(\gamma-1) \delta_{0}^{\gamma-1}},
$$

where we used the fact that $\varepsilon>0$ and is small. To have $v_{0}$ close to $c$ we have to assume either $T_{0}$ large, or $\delta_{0}$ small or $\gamma$ close to 1 .

Let us consider $A_{\bar{n}}=c \sum_{i=1}^{3} A^{i}(z) n_{i}$, where $c$ is the speed of light, and $\bar{n}$ the unit outward normal vector to $\partial \Omega$. The matrix $A_{\bar{n}}$ has the form

$$
A_{\bar{n}}=c\left(\begin{array}{cccc}
u_{n} s_{p} T_{p} & n_{1} & n_{2} \\
n_{1} & u_{n} w\left(1-\beta^{2} u_{1}^{2}\right) & -\beta^{2} u_{n} w u_{1} u_{2} & \\
n_{2} & -\beta^{2} u_{n} w u_{1} u_{2} & u_{n} w\left(1-\beta^{2} u_{2}^{2}\right) \\
n_{3} & -\beta^{2} u_{n} w u_{1} u_{3} & -\beta^{2} u_{n} w u_{2} u_{3} \\
u_{n} s_{p} T_{\delta} & 0 & 0 & u_{n} s_{p} T_{\delta} \\
& n_{3} & 0 \\
& -\beta^{2} u_{n} w u_{1} u_{3} & 0 \\
-\beta^{2} u_{n} w u_{2} u_{3} & 0 \\
u_{n} w\left(1-\beta^{2} u_{3}^{2}\right) & u_{n} T_{\delta}\left(s_{\delta}-s / \delta\right)
\end{array}\right) .
$$

From (6.5) we have

(6.6) $\operatorname{det}\left(-A_{\bar{n}}-\lambda I\right)$

$=-c^{5}\left(u_{n} w+\lambda^{\prime}\right)\left\{\left(u_{n} T_{\delta}\left(s_{\delta}-s / \delta\right)+\lambda^{\prime}\right)\left[\left(u_{n} s_{p} T_{p}+\lambda^{\prime}\right)\left(u_{n} w+\lambda^{\prime}\right)\left(u_{n} w \beta^{2}+\lambda^{\prime}\right)\right.\right.$ 


$$
\left.\left.-\left(u_{n} w \beta^{2}\left(u_{n}^{2}+1\right)+\lambda^{\prime}\right)\right]-\left(u_{n} s_{p} T_{\delta}\right)^{2}\left(u_{n} w+\lambda^{\prime}\right)\left(u_{n} w \beta^{2}+\lambda^{\prime}\right)\right\}
$$

where $\lambda^{\prime}=\lambda / c$ so $\lambda_{1}=-c u_{n} w$.

By local straightening of the boundary (given by the transformation $T: y=y(x)$, see $4(\mathrm{c}))$, we can assume

$$
u_{n}^{2}=\left(u_{1} n_{1}+u_{2} n_{2}+u_{3} n_{3}\right)^{2}=u_{1}^{2}+u_{2}^{2}+u_{3}^{2}=1 / \beta^{2}-1 .
$$

Therefore we get

$$
\operatorname{det}\left(-A_{\bar{n}}-\lambda I\right)=\left(c u_{n} w+\lambda\right)^{2}\left(\lambda^{3}+c b \lambda^{2}-c^{2} a \lambda+c^{3} d\right)
$$

where

$$
\begin{aligned}
a= & \left(u_{n} s_{p} T_{\delta}\right)^{2}-u_{n}^{2} T_{\delta}\left(s_{\delta}-s / \delta\right) s_{p} T_{p} \\
& -u_{n}^{2} T_{\delta}\left(s_{\delta}-s / \delta\right) w \beta^{2}-u_{n}^{2} s_{p} T_{p} w \beta^{2}+1, \\
b= & u_{n} T_{\delta}\left(s_{\delta}-s / \delta\right)+u_{n} s_{p} T_{p}+u_{n} w \beta^{2}, \\
d= & -\left(u_{n} s_{p} T_{\delta}\right)^{2} u_{n} w \beta^{2}-u_{n} T_{\delta}\left(s_{\delta}-s / \delta\right) \\
& +u_{n}^{3} T_{\delta}\left(s_{\delta}-s / \delta\right) s_{p} T_{p} w \beta^{2} .
\end{aligned}
$$

We examine the polynomial

$$
f(\lambda)=\lambda^{3}+c b \lambda^{2}-c^{2} a \lambda+c^{3} d
$$

with derivative

$$
f^{\prime}(\lambda)=3 \lambda^{2}+2 c b \lambda-c^{2} a .
$$

Using the solutions of $f^{\prime}(\lambda)=0$ :

$$
x_{1}=-c \frac{b+\sqrt{b^{2}+3 a}}{3}, \quad x_{2}=c \frac{-b+\sqrt{b^{2}+3 a}}{3},
$$

we can calculate the local maximum $f\left(x_{1}\right)$ and minimum $f\left(x_{2}\right)$ of $f(\lambda)$; next, solving $f(x)-f\left(x_{1}\right)=0$ and $f(x)-f\left(x_{2}\right)=0$ we find $x_{r}$ and $x_{l}$, respectively, such that

$$
x_{l}<\lambda_{3} \leq x_{1} \leq \lambda_{4} \leq x_{2} \leq \lambda_{5}<x_{r}
$$

where $\lambda_{3}, \lambda_{4}, \lambda_{5}$ are the roots of the second term of the characteristic polynomial (6.6).

Moreover, for $\lambda_{1}=\lambda_{2}=-c u_{n} w$ we have

$$
u_{n} \leq 3\left(2 / \varrho^{2}-1+6 \varepsilon^{2}\right), \quad w \leq w_{0}+\varepsilon .
$$

Hence we formulate

LEMMA 6.2. Let the assumptions of Lemma 6.1 be satisfied and additionally suppose that

(a) $s_{p}, T_{p}, T_{\delta}, s_{\delta}-s / \delta$ are bounded,

(b) $d=-\left(u_{n} s_{p} T_{\delta}\right)^{2} u_{n} w \beta^{2}-u_{n} T_{\delta}\left(s_{\delta}-s / \delta\right)+u_{n}^{3} T_{p} s_{p} T_{\delta}\left(s_{\delta}-s / \delta\right) w \beta^{2} \neq 0$. 
Then the eigenvalues $\lambda_{i}$ of the matrix $-A_{\bar{n}}=-c \sum_{i=1}^{3} A^{i}(z) \bar{n}_{i}$ and the matrix $E=A^{0}(z)$ satisfy conditions (a) and (c) of Section 5 and Theorem 5.1.

Now we are finally prepared to formulate the result:

THEOREM 6.1. Suppose the following assumptions are satisfied:

(1) $g \in H_{\alpha}^{3}\left(\partial \Omega^{t}\right), z_{0} \in H^{4}(\Omega),\left.z_{0}\right|_{\partial \Omega}=0$.

(2) $\partial \Omega \in C^{3}$.

(3) For $z_{0}=\left(p_{0}, u_{01}, u_{02}, u_{03}, \delta_{0}\right)$ we have

(a) $p_{0}>0, \delta_{0}>0$

(b) $v_{0}^{2}=v_{01}^{2}+v_{02}^{2}+v_{03}^{2} \leq\left(1-\varrho^{2}\right) c^{2}$, where $\varrho \in(0,1)$.

(4) $s_{p}, T_{p}, T_{\delta}, s_{\delta}-s / \delta$ are bounded and of the same sign.

(5) $d \neq 0$ (see (6.7) or Lemma 6.2).

(6) The matrix $M(x, t, z)$ has the form described in 5.1(b),

(7) The matrices $E(t, x, z(x, t)), A_{i}(t, x, z(x, t))=c A^{i}(t, x, z(x, t))$ are 3 -times differentiable functions with respect to $t, x, z$.

(8) $\|g\|_{3, \partial \Omega^{t}, \alpha}$ and $\left\|z_{0, x}\right\|_{3,2, \Omega}$ are sufficiently small, and $t \leq t^{*}$ (see Section 5).

(9) $\alpha$ satisfies $\alpha \alpha_{0} \geq \widehat{p}_{0}\left(Q, d+\left\|z_{0}\right\|_{3,2, \Omega}\right) \geq p_{0}(a, b)+c a^{2}$ for some polynomials $\widehat{p}_{0}, p_{0}$ and $Q=Q\left(G_{0}, \varepsilon\right)$ is defined in Section 5 .

Then there exists a solution of (2.2) such that

$$
z \in \Pi_{0}^{3}\left(\Omega^{t}\right) \cap H_{\alpha}^{3}\left(\Omega^{t}\right) \cap H_{\alpha}^{3}\left(\partial \Omega^{t}\right) .
$$

Moreover, under the assumptions

$$
\begin{gathered}
\sup _{Q} \sup _{\Omega^{t}}\left|\widetilde{L}^{\prime}(z)\right| \sup _{\Omega^{t}}\left(\left|z_{1}\right|+\left|D_{t, x}^{1} z_{1}\right|\right) \leq\left(\alpha \alpha_{0}\right)^{2} / 8 \\
\left(c_{0}+c_{1}\right) \delta_{0}^{-2} \sup _{Q} \sup _{\partial \Omega^{t}}\left|M^{\prime}(z)\right| \sup _{\Omega^{t}}\left|z_{1}\right| \leq c_{0} / 4
\end{gathered}
$$

for some solutions $z_{1} \in C^{1}\left(\Omega^{t}\right)$ we have uniqueness.

REMARK. Introducing the quantity $z-z_{0}$ in the method of successive approximations, we have avoided the assumption that $z_{0}$ is small. We need, in fact, the smallness of $z_{0, x}$ in $H^{3}$ and of $g(t, x)$ in $H_{\alpha}^{3}\left(\partial \Omega^{t}\right)$. That is very important in the relativistic case, where the condition $\left|z_{0}\right|<1$ means that $v_{0}^{2}<c^{2} / 2$, which is very restrictive.

7. Barotropic case. We additionally consider the problem (1.1)-(1.2) in the barotropic case (that means, the pressure $p$ is an explicit function of the density $\delta$ ). As before, $p$ and $\delta$ denote variables as measured in the reference frame moving with the fluid.

We assume that

$$
w=\delta c^{2}+\delta e_{0}+p
$$




$$
p=\delta^{2} \frac{\partial e_{0}}{\partial \delta},
$$

where $e_{0}$ is the specific internal energy $e_{0}=e_{0}(\delta)$.

We can write equations (1.1)-(1.2) in the form

$$
\begin{gathered}
\frac{\partial}{\partial x^{k}}\left[\delta\left(c^{2}+e_{0}\right)+p\right] u_{i} u^{k}+\left[\delta\left(c^{2}+e_{0}\right)+p\right] \frac{\partial}{\partial x^{k}}\left(u_{i} u^{k}\right)+\frac{\partial}{\partial x_{i}} p=0, \\
\frac{\partial}{\partial x^{i}}\left(\delta u^{i}\right)=0 .
\end{gathered}
$$

Notice that we now have 5 equations and 4 unknowns (because $p$ is given by (7.2)). Moreover, it is easier to find $\lambda^{i}(\underline{z})$, where $\underline{z}=\left(u^{1}, u^{2}, u^{3}, \delta\right)$, such that $\lambda^{i}$ are the coefficients of linear dependence for equations (7.3)-(7.4). By multiplying (7.3) by $u^{i}$ and summing over $i$ we get

$$
-\frac{\partial}{\partial x^{k}}\left[\delta\left(c^{2}+e_{0}\right)+p\right] u^{k}-\left[\delta\left(c^{2}+e_{0}\right)+p\right] \frac{\partial u^{k}}{\partial x^{k}}+\frac{\partial p}{\partial x^{i}} u^{i}=0 .
$$

This implies

$$
-\left(c^{2}+e_{0}\right) \frac{\partial \delta}{\partial x^{k}} u^{k}-\left(c^{2}+e_{0}\right) \delta \frac{\partial u^{k}}{\partial x^{k}}-\delta \frac{\partial e_{0}}{\partial x^{k}} u^{k}-p \frac{\partial u^{k}}{\partial x^{k}}=0 .
$$

Using (7.2) we get

$$
-\left(c^{2}+e_{0}\right) \frac{\partial}{\partial x^{k}}\left(\delta u^{k}\right)-\delta \frac{\partial e_{0}}{\partial \delta}\left(\frac{\partial \delta}{\partial x^{k}} u^{k}+\delta \frac{\partial u^{k}}{\partial x^{k}}\right)=0
$$

so adding the equation of continuity (7.4) with multiplier $\lambda^{4}=c^{2}+e_{0}+$ $\delta \partial e_{0} / \partial \delta \equiv c^{2}+e_{0}+p / \delta \equiv w / \delta$ to $(7.5)$ we obtain zero.

In this way we have found $\lambda^{m}=\left(u^{0}, u^{1}, u^{2}, u^{3}, w / \delta\right)$ for system (7.3)(7.4). We calculate

$$
\partial_{\underline{z}^{\tau}} \lambda^{m}=\left(\begin{array}{ccccc}
\beta u_{1} & 1 & 0 & 0 & 0 \\
\beta u_{2} & 0 & 1 & 0 & 0 \\
\beta u_{3} & 0 & 0 & 1 & 0 \\
0 & 0 & 0 & 0 & \frac{1}{\delta} \frac{\partial p}{\partial \delta}
\end{array}\right)
$$

because

$$
\frac{\partial}{\partial \delta}\left(\frac{w}{\delta}\right)=\frac{\partial e_{0}}{\partial \delta}+\frac{\partial}{\partial \delta}\left(\delta \frac{\partial e_{0}}{\partial \delta}\right)=2 \frac{\partial e_{0}}{\partial \delta}+\delta \frac{\partial^{2} e_{0}}{\partial \delta^{2}}=\frac{1}{\delta} \frac{\partial p}{\partial \delta} .
$$

Rewriting (7.3)-(7.4) in the form

$$
\partial_{\underline{z}^{j}} q_{m}^{k}(\underline{z}) \frac{\partial \underline{z}^{j}}{\partial x^{k}}=0, \quad m=0, \ldots, 4,
$$

we obtain

$$
B_{\tau_{j}}^{k} \frac{\partial \underline{z}^{j}}{\partial x^{k}}=0 \quad \text { where } \quad B_{\tau_{j}}^{k}=\partial_{\underline{z}^{\tau}} \lambda^{m} \partial_{\underline{z}^{j}} q_{m}^{k}(\underline{z}), i, k, j=0, \ldots, 3,
$$

and $B^{k}(\underline{z})$ are symmetric. 
Let us consider $B^{0}(\underline{z})$ and the condition $B^{0} \underline{z} \cdot \underline{z}>\alpha_{0} \underline{z}^{2}$. We calculate

$$
\partial_{\underline{z}^{j}} q_{m}^{0}(\underline{z})=\left(\begin{array}{cccc}
-2 w u_{1} & -2 w u_{2} & -2 w u_{3} & p_{\delta}-\frac{1}{\beta^{2}} \frac{w}{\delta} \\
\frac{1}{\beta} w+\beta w u_{1}^{2} & \beta w u_{1} u_{2} & \beta w u_{1} u_{3} & \frac{1}{\beta} \frac{w}{\delta} u_{1} \\
\beta w u_{1} u_{2} & \frac{1}{\beta} w+\beta w u_{2}^{2} & \beta w u_{2} u_{3} & \frac{1}{\beta} \frac{w}{\delta} u_{2} \\
\beta w u_{1} u_{3} & \beta w u_{2} u_{3} & \frac{1}{\beta} w+\beta w u_{3}^{2} & \frac{1}{\beta} \frac{w}{\delta} u_{3} \\
\beta \delta u_{1} & \beta \delta u_{2} & \beta \delta u_{3} & \frac{1}{\beta}
\end{array}\right)
$$

and multiplying by $\partial_{\underline{z}^{\tau}} \lambda^{m}$ gives

$$
B^{0}(\underline{z})=\left(\begin{array}{cccc}
-\beta w u_{1} u_{1}^{2}+\frac{w}{\beta} & -\beta w u_{1} u_{2} & -\beta w u_{1} u_{3} & \beta p_{\delta} u_{1} \\
-\beta w u_{1} u_{2} & -\beta w u_{2}^{2}+\frac{w}{\beta} & -\beta w u_{2} u_{3} & \beta p_{\delta} u_{2} \\
-\beta w u_{1} u_{3} & -\beta w u_{2} u_{3} & -\beta w u_{3}^{2}+\frac{w}{\beta} & \beta p_{\delta} u_{3} \\
\beta p_{\delta} u_{1} & \beta p_{\delta} u_{2} & \beta p_{\delta} u_{3} & \frac{1}{\beta} \frac{p_{\delta}}{\delta}
\end{array}\right)
$$

so we find

$$
\begin{aligned}
B^{0} \underline{z} \cdot \underline{z} & \left(\begin{array}{c}
\beta w u_{1}\left(\frac{1}{\beta^{2}}-u_{1}^{2}-u_{2}^{2}-u_{3}^{2}\right)+\beta p_{\delta} \delta u_{1} \\
\beta w u_{2}\left(\frac{1}{\beta^{2}}-u_{1}^{2}-u_{2}^{2}-u_{3}^{2}\right)+\beta p_{\delta} \delta u_{2} \\
\beta w u_{3}\left(\frac{1}{\beta^{2}}-u_{1}^{2}-u_{2}^{2}-u_{3}^{2}\right)+\beta p_{\delta} \delta u_{3} \\
\beta p_{\delta}\left(u_{1}^{2}+u_{2}^{2}+u_{3}^{2}+\frac{1}{\beta^{2}}\right)
\end{array}\right)\left(\begin{array}{c}
u_{1} \\
u_{2} \\
u_{3} \\
\delta
\end{array}\right) \\
= & \beta w\left(u_{1}^{2}+u_{2}^{2}+u_{3}^{3}\right)+2 \beta p_{\delta} \delta\left(u_{1}^{2}+u_{2}^{2}+u_{3}^{2}\right)+\frac{p_{\delta}}{\beta} \delta .
\end{aligned}
$$

Using (see Lemma 6.1) $\beta \geq\left(2 / \varrho^{2}-1+6 \varepsilon^{2}\right)^{-1}, w \geq p_{0}-\varepsilon$ and $\partial p / \partial \delta>0$, $\delta>0$ we have

$$
B^{0} \underline{z} \cdot \underline{z} \geq\left(2 / \varrho^{2}-1+6 \varepsilon^{2}\right)^{-1}\left(p_{0}-\varepsilon\right)\left(u_{1}^{2}+u_{2}^{2}+u_{3}^{2}\right)+\frac{p_{\delta}}{\delta} \delta^{2} .
$$

Because $\partial p / \partial \delta>0$ we can find some constant $\widetilde{c}$ such that $\partial p / \partial \delta>\widetilde{c}>0$; as $\delta \leq \delta_{0}+\varepsilon$ we hence obtain

$$
\begin{aligned}
B^{0} \underline{z} \cdot \underline{z} \geq & \left(2 / \varrho^{2}-1+6 \varepsilon^{2}\right)^{-1}\left(p_{0}-\varepsilon\right)\left(u_{1}^{2}+u_{2}^{2}+u_{3}^{2}\right) \\
& +\frac{\widetilde{c}}{\delta_{0}+\varepsilon} \delta^{2} \leq \underline{\alpha}_{0} \underline{z}^{2}
\end{aligned}
$$

where

$$
\underline{\alpha}_{0}=\min \left\{\left(2 / \varrho^{2}-1+6 \varepsilon^{2}\right)^{-1}\left(p_{0}-\varepsilon\right), \widetilde{c} /\left(\delta_{0}+\varepsilon\right)\right\} .
$$

\section{References}

[1] K. O. Friedrichs, Conservation equations and laws of motion in classical physics, Comm. Pure Appl. Math. 31 (1978), 123-131.

[2] - - On the laws of relativistic electromagnetofluid dynamics, ibid. 27 (1974), 749808. 
[3] K. O. Friedrichs and P. D. Lax, Boundary value problem for the first order operators, ibid. 18 (1965), 355-388.

[4] L. Landau and E. Lifschitz, Hydrodynamics, Nauka, Moscow, 1986 (in Russian); English transl.: Fluid Mechanics, Pergamon Press, Oxford, 1987.

[5] P. D. Lax and R. S. Phillips, Local boundary conditions for dissipative symmetric linear differential operators, Comm. Pure Appl. Math. 13 (1960), 427-455.

[6] S. Mizohata, Theory of Partial Differential Equations, Mir, Moscow, 1977 (in Russian).

[7] M. Nagumo, Lectures on Modern Theory of Partial Differential Equations, Moscow, 1967 (in Russian).

[8] J. Smoller and B. Temple, Global solutions of the relativistic Euler equations, Comm. Math. Phys. 156 (1993), 67-99.

[9] W. M. Zajączkowski, Non-characteristic mixed problems for non-linear symmetric hyperbolic systems, Math. Meth. Appl. Sci. 11 (1989), 139-168.

[10] -, Non-characteristic mixed problem for ideal incompressible magnetohydrodynamics, Arch. Mech. 39 (1987), 461-483.

Joanna Rencławowicz and Wojciech M. Zajączkowski

Institute of Mathematics

'Sniadeckich 8

00-950 Warszawa, Poland

E-mail: jr@impan.gov.pl

wmzajacz@impan.gov.pl

Received on 14.4.1997;

revised version on 17.10 .1997 